\title{
Coupling of terminal differentiation deficit with neurodegenerative pathology in Vps35-deficient pyramidal neurons
}

\author{
Fu-Lei Tang ${ }^{1} \cdot$ Lu Zhao ${ }^{1,2,3} \cdot$ Yang Zhao ${ }^{2,3} \cdot$ Dong Sun ${ }^{1,2} \cdot$ Xiao-Juan Zhu ${ }^{3} \cdot$ Lin Mei ${ }^{1,2} \cdot$ Wen-Cheng Xiong ${ }^{1,2}$
}

Received: 20 June 2019 / Revised: 13 December 2019 / Accepted: 17 December 2019 / Published online: 6 January 2020

(c) The Author(s), under exclusive licence to ADMC Associazione Differenziamento e Morte Cellulare 2020. This article is published with open access

\begin{abstract}
Vps35 (vacuolar protein sorting 35) is a key component of retromer that regulates transmembrane protein trafficking. Dysfunctional Vps35 is a risk factor for neurodegenerative diseases, including Parkinson's and Alzheimer's diseases. Vps35 is highly expressed in developing pyramidal neurons, and its physiological role in developing neurons remains to be explored. Here, we provide evidence that Vps35 in embryonic neurons is necessary for axonal and dendritic terminal differentiation. Loss of Vps35 in embryonic neurons results in not only terminal differentiation deficits, but also neurodegenerative pathology, such as cortical brain atrophy and reactive glial responses. The atrophy of neocortex appears to be in association with increases in neuronal death, autophagosome proteins (LC3-II and P62), and neurodegeneration associated proteins (TDP43 and ubiquitin-conjugated proteins). Further studies reveal an increase of retromer cargo protein, sortilin1 (Sort1), in lysosomes of Vps35-KO neurons, and lysosomal dysfunction. Suppression of Sort1 diminishes Vps35KO-induced dendritic defects. Expression of lysosomal Sort1 recapitulates Vps35-KO-induced phenotypes. Together, these results demonstrate embryonic neuronal Vps35's function in terminal axonal and dendritic differentiation, reveal an association of terminal differentiation deficit with neurodegenerative pathology, and uncover an important lysosomal contribution to both events.
\end{abstract}

\section{Introduction}

Neurodegenerative diseases, including Alzheimer's disease (AD), Parkinson's disease (PD), and frontotemporal dementia (FTD), occur as a result of complex brain degenerative processes with a progressive loss of neurons or brain structures [1]. Among numerous risk factors for

Edited by N. Bazan

Supplementary information The online version of this article (https:// doi.org/10.1038/s41418-019-0487-2) contains supplementary material, which is available to authorized users.

$\triangle$ Wen-Cheng Xiong

Wen-Cheng.Xiong@case.edu

1 Department of Neuroscience and Regenerative Medicine, Medical College of Georgia, Augusta University, Augusta 30912, Georgia

2 Department of Neurosciences, Case Western Reserve University, School of Medicine, Cleveland, OH 44106, USA

3 Key Laboratory of Molecular Epigenetics of Ministry of Education, Institute of Cytology and Genetics, Northeast Normal University, Changchun, Jilin 130024, China neurodegenerative diseases, genetic mutations are believed to be a primary "hit" [2]. While each disease has its unique risk genes, mutations in VPS35 have been associated with PD and AD [3-5], suggesting that Vps35 dysfunction may be a general risk factor for neurodegenerative disorders.

Vps35 is a key component of retromer that selectively sorts transmembrane proteins/cargos to the trans-Golgi network or plasma membrane [6-8]. Several lines of evidence suggest that Vps35 dysfunction is linked with PD and AD. Vps35 is reduced in the hippocampus of AD patients [9]. Vps35-deficiency increases production of $A \beta$ [9-11] and impairs mitochondrial dynamics and function $[12,13]$. Vps35-deficient mice exhibit partial AD- or PD-relevant deficits [10, 11, 14] and overexpressing Vps35 fully recovers the AD phenotype in $3 \times \mathrm{Tg}$ mice [15]. In addition, retromer cargo proteins including APP [16], TREM2 [17], and sortilin1-related receptor (SorLA) [18] are genetic risk factors for AD. Interestingly, sortilin1 (Sort1) and SorLA are members of vacuolar protein sorting ten family receptors. Sort1 has been emerged as a co-receptor in cell death or neurodegeneration regulated by ligands of progranulin (PGRN) and implicated in the pathogenesis of FTD [19]. 
These observations implicate that Vps35-deficiency may be a common pathological mechanism of neurodegenerative disorders, including AD, PD, and possibly FTD. However, how Vps35-loss results in different neurodegenerative disorders and whether Vps35-loss increases FTD development remains largely unknown.

Vps35 is highly expressed in developing pyramidal neurons [20] as well as dopamine (DA) neurons [14]. We have previously shown that selectively knocking out (KO) Vps35 in DA neurons results in early onset PD-relevant deficits [12]. Here, we investigated Vps35's function in mouse developing pyramidal neurons. $\mathrm{Vps} 35-\mathrm{KO}$ in embryonic cortical pyramidal neurons results in dendritic maturation defects and axonal spheroid formation. Mice that selectively depleted Vps35 gene in embryonic (by Neurod6-Cre) pyramidal neurons display FTD-like neuropathology, including progressive reduction of cortical thickness, elevation of cortical neuronal death, accumulations of P62, LC3-II, Tdp43, and ubiquitin-conjugated protein levels, impairments in lysosomal morphology and acidification, and reactive gliosis. Further mechanical studies demonstrate an increase of Sort1 in lysosomal compartments of Vps35-KO neurons. Suppression of Sort1 expression by shRNA diminishes Vps35-loss-induced dendritic defects. Expression of lysosomal Sort1 fusion protein in embryonic pyramidal neurons impairs lysosomal functions and recapitulates Vps35-loss-induced deficits. These observations thus uncover Sort1 as a critical cargo of Vps35 to underlie Vps35's function in developing neurons. Our results suggest that the dysfunctional Vps35-Sort1 pathway in developing pyramidal neurons acts as a detrimental factor not only for neuronal terminal differentiation but also for neurodegenerative pathology.

\section{Methods and materials}

\section{Animals}

Mice were cared according to animal protocols approved by the Institute of Animal Care and Use Committees at the Augusta University and Case Western Reserve University, according to the National Institute of Health (NIH) guidelines. All mice were housed in standard conditions with food and water provided and maintained on a $12 \mathrm{~h}$ dark/ light cycle. The noon of a day when a vaginal plug is found is designated as E0.5. Experiments were replicated at a minimum of three times with mice derived from independent litters. Vps35 loxp flanked exon 6 mice have been previously described [12]. Vps35-cKO mice were generated by breeding floxed Vps35 (Vps35fff) with Neurod6-Cre (kindly provided by Dr KA Nave [21]) mice. Neurod6-Cre expression alone did not have a detectable effect on the phenotypes described in this manuscript. Thus, the Crepositive or Cre-negative littermates were utilized as controls unless indicated otherwise. Ai9 (stock No. 007907) and Thyl-YFP (stock No. 003782) mice were purchased from the Jackson laboratories. For all experiments, mutants were compared with littermate controls. All phenotypic characterizations of Vps35-cKO (Vps35 $5^{\text {Neurod6 }}$ ) and their control mice were in C57BL/6 background. Both male and female mice were used in all experiments.

\section{Plasmids}

The $p C A G-G F P$ and $p C A G$-Cre plasmids were purchased from Addgene. To generate the pCAG-Cre-2A-GFP or BFP plasmid, the $P 2 A$ domain was added to the $C$ terminus of Cre, then followed by $G F P$ or $B F P$ cDNA. Their Cre efficiency was confirmed by IUE of the plasmid into Ai9 reporter mice.

The Sort1-ShR expression vector was generated by the pSuper vector system, the target sequence is $5^{\prime}$-gcacctga caacaaatggg (Sort1 shR2\#) and 5'-ccgtcctatcaatgtgatt (Sort1 shR 3\#).

To generate Lyso-Sort1 (CD63-Sort1) plasmid, the CD63 cDNA was cloned from mouse whole brain mRNA by TA cloning. Sort1 cDNA (without the signal peptide) was then inserted into the first luminal loop of CD63 by Ligation Independent Cloning (LIC) with Exonuclease III. Finally, the OFR with CD63-Sort1 were inserted into a mammalian expression vector with a $C A G$ promoter. To test the $\mathrm{pH}$ in lysosomes, Lyso-pHluorin (Plasmid \#70113) were obtained via Addgene, and the promoter $\mathrm{CMV}$ was replaced with CAG.

\section{In utero electroporation (IUE)}

IUE was performed as previously described [22]. In brief, pregnant mice were deeply anesthetized with $2 \%$ isoflurane (Piramal Healthcare) through anesthesia system (Kent Scientific), and embryos were exposed at E14.5 or E18.5. The plasmid (at a final concentration of $\sim 1 \mu \mathrm{g} / \mu \mathrm{l}$ ) was mixed with fast green $(0.1 \mathrm{mg} / \mathrm{ml}$, Sigma-Aldrich), which were microinjected into the lateral ventricle of embryos using a glass capillary. The embryos were electroporated with five $50 \mathrm{~ms}$ pulses at $36 \mathrm{~V}$ (for E14.5) or $45 \mathrm{~V}$ (for E18.5) with a $950 \mathrm{~ms}$ interval through ECM-830 (BTX, Holliston, MA). The uterine horns were then gently reinserted into the abdominal cavity and the abdomen wall and skin were sutured. The pups were fixed by cardiac perfusion with $4 \%$ paraformaldehyde in $0.1 \mathrm{M}$ phosphate buffer, $\mathrm{pH} 7.4$. at different postnatal days. Their brains were removed and soaked in the fixative for 2-4h. After rinsing with phosphate-buffered saline (PBS), their coronal vibratome sections $(50-100 \mu \mathrm{m}$ in thickness) were prepared by 
(VT1000s, Leica) and used for immunostaining or immunohistochemical staining analyses.

\section{Antibodies for immunohistochemistry and immunoblotting}

The following antibodies were used: Mouse monoclonal anti-beta-Actin (diluted 1:5,000; Abcam, ab8227); goat polyclonal anti-aldolase C (diluted 1:200; Santa Cruz, sc12065); rabbit monoclonal anti-CD11b (diluted 1:200; Abcam, ab133357); rabbit monoclonal anti-cleaved caspase-3 (Asp175) (D3E9) (diluted 1:400; Cell Signaling, 9579); mouse monoclonal anti-GM130 (diluted 1:1000; BD Bioscience, 610822); mouse monoclonal anti-GFAP (1:500; Millipore, MAB360); rabbit polyclonal anti-GFP (1:2,000; Thermo Fisher Scientific, A-11122); rabbit polyclonal antiEEA1 (diluted 1:5000; Abcam, ab50313); goat polyclonal Iba1 (diluted 1:500; Abcam, ab5076); rat monoclonal antiLamp1 (1:500; DSHB, 1d4b); rabbit polyclonal anti-LC3B (diluted 1:500; Abcam, ab48394); mouse monoclonal antiNeuN (diluted 1:1000; EMD Millipore, MAB377); mouse monoclonal anti-P53 (diluted 1:800; abcam, ab26); Sheep polyclonal anti-PGRN (diluted 1:500; R\&D Systems, AF2557); rabbit polyclonal anti-Sort1 (diluted 1:500; Abcam, ab16640); mouse monoclonal anti-P62 (diluted 1:500; Abcam,) ab56416, rabbit polyclonal anti-TDP43 (Cterminal) (diluted 1:500; Proteintech, 12892-1-AP,); rat monoclonal anti-phospho-TDP43 (Ser409/Ser410) (diluted 1:100; Millipore Sigma, MABN14); mouse monoclonal anti-ubiquitin (diluted 1:500; Santa Cruz, sc-8017); mouse monoclonal anti- $\gamma \mathrm{H} 2 \mathrm{AX}$ (phosphorylation of the Ser-139 residue of the histone variant $\mathrm{H} 2 \mathrm{AX}$ ) (diluted 1:500; abcam, ab26350); rabbit polyclonal anti-Vps35 and rabbit polyclonal anti-reticulum 3 (Rtn3) antibody was generated as described previously [10, 23] (diluted 1:2000).

\section{Immunohistochemistry}

Mice of the appropriate age were anesthetized and perfused transcardially with $4 \%$ paraformaldehyde/PBS as previously described [10, 12, 14]. Brains were dissected, postfixed, and mounted in agarose prior to vibratome sectioning. Overall, 35-100 $\mu \mathrm{m}$ free-floating vibratome sections of brains were collected in PBS, blocked with 5\% normal serum in PBS with $0.5 \%$ Triton X-100 and incubated with primary antibodies in blocking solution overnight at $4{ }^{\circ} \mathrm{C}$. P14 vibratome sections were blocked with $5 \%$ normal serum in PBS with $0.1 \%$ Triton X-100 and $2 \%$ DMSO, and incubated with primary antibodies for 3 days. After 1-3 h incubation with Alexa Fluor-conjugated secondary antibodies (Invitrogen or Jackson Immunoresearch) followed by DAPI staining $(0.1 \mu \mathrm{g} / \mathrm{ml}$, Life Technologies), the stained slices were imaged using a confocal laser- scanning microscope (Zeiss LSM510, LSM710 or Nikon A1 plus).

\section{Image acquisition and analysis}

Confocal images of regions of interest were collected from individual brain sections for each animal. For all morphological analyses, images were coded using computergenerated random number sequences at the time of data acquisition and all analyses were performed blinded to the experimental condition. Control and experimental group neurons which were to be directly compared were imaged with the same acquisition parameters.

Z-stack images were collected with $\times 10$ or $\times 20$ objectives and tiled together to generate high-resolution images of whole brain sections. The acquired images were processed using the Zen (Zeiss) Representative images have been cropped and adjusted for brightness and contrast in Photoshop for presentation. For the quantitative analysis of intracellular levels of P62, p-TDP43, Sort1 and pHluorin, the entire neuron was selected, and the fluorescence intensity was measured directly with Image $\mathbf{J}$ after a threshold application.

\section{Dendritic arborization}

Individual neurons from Thyl-YFP mice or IUEed brain were acquired on a Nikon A1 plus confocal system using a W-Plan Apochromat 25×/1.0 IR-corrected water immersion objective. On average, Z-stacks were acquired from 50 to $70 \mu \mathrm{m}$ range with $1 \mu \mathrm{m}$ steps. Dendritic reconstructions were performed using NeuronStudio. A total of 12 control and 12 experimental neurons from three mice each were reconstructed for dendritic analysis. Apical and basal dendrite polarity was quantified by NeuronJ from reconstructed images. Sholl analysis calculated the number of dendrite intersections and dendritic lengths as previously described [24]. Sholl quantification was conducted with an initial $10-\mu \mathrm{m}$ soma radius and $10-\mu \mathrm{m}$ concentric radial steps.

\section{Dendritic spines and microglia imaging and 3D reconstructions}

For quantitative analysis of the morphology of dendrite spines and dendrites. Images of dendritic spines/microglia were acquired on a Nikon A1 confocal microscope with a Plan Apo VC 60 X Oil DIC N2 objective (N.A. = 1.4), at 3X zooms, $0.5 \mathrm{um}$ Z-interval, and $1024 \times 1024$ pixels. Three-dimensional (3D) reconstructions were performed, and spine density was quantified using the Filament module of IMARIS software (Bitplane) as described previously [25, 26].

For the spine/dendrite ratio analysis, secondary branches with similar width of apical dendrites of L2-3 
pyramidal neurons in the somatosensory cortex were examined. Spine density was calculated by dividing the total spine number by the dendritic branch length. Spine subtypes were classified based on previously defined morphological criteria [25] and quantitated as follows: (a) mushroom: the spine head diameter is $\geq 1.5 \times$ the diameter of spine neck ( $\mathrm{dh} / \mathrm{dn} \geq 1.5)$; (b) stubby: head and neck of the spine are approximately of same width, and spine length is not significantly longer than the head diameter $(\mathrm{dh} / \mathrm{dn}<1.5, \mathrm{~L} / \mathrm{dh}<2)$; (c) thin: diameters of spine head and neck are nearly equal, and spine length is greater than spine width $(\mathrm{dh} / \mathrm{dn}<1.5, \mathrm{~L} / \mathrm{dh} \geq 2)$; Only images (blindcoded) with sufficient quality to clearly identify and measure spine shapes were used in the subtype classification, and 20-30 consecutive spines on the same dendrite were analyzed per image.

\section{Nissl staining, Golgi staining, and TUNEL assay}

For Nissl staining sections were incubated in $1 \%$ cresyl violet solution for 3-4 min, then washed and dehydrated in increasing ethanol concentrations. The cortical thickness was measured in Nissl-stained sections using ImageJ software. For each animal, we took three measurements in the somatosensory cortex and the visual cortex $(n=3-4$ animals per genotype). Golgi staining was performed by using the FD Rapid GolgiStain Kit (FD NeuroTechnologies) as described previously [27]. Freshly dissected brain was immersed in the kit's impregnation solution for 2 weeks. The frozen tissue was sectioned at $150 \mu \mathrm{m}$ on a microtome, followed by staining according to the manufacturer's procedures. For the neuronal morphometric analysis, pyramidal neurons were randomly selected from somatosensory cortical layer II/III. Images were acquired ( 2 or $0.2 \mu \mathrm{m}$ pitch) using a light microscope at $\times 10$ or $\times 40$ magnification (BX43; Olympus), and the dendrites and dendritic spines were traced using NeuronStudio software. The total dendritic length, Sholl analysis, and spine density were also calculated by Neuron $\mathbf{J}$ (ImageJ). The spinal density was obtained from the apical dendrites within the region 50-150 $\mu \mathrm{m}$ from the soma (total length 1300-1800 $\mu \mathrm{m}$ ). The TUNEL assay was used to detect dead cells with DNA fragmentation using Click-iT Plus TUNEL assay (Invitrogen) by following manufacture's protocol.

\section{NeuroSilver Staining}

Silver staining was used to visualize degenerating neuronal elements in brain sections of $\mathrm{Vps} 35^{\text {Neurod6 }}$ mice and agematched control mice [28]. Sections were processed using the FD NeuroSilver kit (FD Neurotechnologies Inc, Baltimore, MD) according to the manufacturer's instructions.

\section{Jade C Staining}

Fluoro-Jade C (Jade C) a polyanionic fluorescein derivative that can sensitively and selectively bind to degenerative neurons [29]. To visualize degenerating neurons, brain sections from perfused P14 WT and Vps $35^{\text {Neurod6 }}$ brains were mounted on gelatin-coated slides and subjected to Jade C staining, according to manufacturer's protocol (FluoroJade $^{\circledR}$ C, Millipore). Sections were imaged with a Zeiss LSM510 confocal microscope.

\section{Western blotting}

Tissues were dissected from both mutant and littermate control mice and lysed with $5 \times$ volume per weight $1 \%$ Triton $\mathrm{X}-100$ buffer (1\% Triton X-100, $20 \mathrm{mM}$ Tris pH 7.4, $150 \mathrm{mM} \mathrm{NaCl}, 10 \%$ glycerol, protease and phosphatase inhibitor cocktail (Millipore)) by homogenization, incubated on ice-water slurry for $20 \mathrm{~min}$, frozen and thawed twice, and ultracentrifuged at $100,000 \mathrm{~g}, 4{ }^{\circ} \mathrm{C}$ for $30 \mathrm{~min}$ as described previously [12] and the supernatant taken as the soluble fraction. The Triton-insoluble pellets were sonicated with $2 \times$ volume per original sample weight in urea-lysis buffer (7 M urea, $2 \mathrm{M}$ thiourea, $4 \%$ CHAPS, and $30 \mathrm{mM}$ Tris, $\mathrm{pH} 7.5$, and a protease and phosphatase inhibitor cocktail (Millipore)) and centrifuged at $100,000 \mathrm{~g}$ for $30 \mathrm{~min}$ at $22^{\circ} \mathrm{C}$. These supernatants were taken as the insoluble fraction. Protein concentration was measured by BCA assay (Pierce).

Lysosomal proteins were isolated as described previously [30]. In brief, neocortex lysates from P1 mice were homogenized in a homogenizing buffer (HM buffer; $0.25 \mathrm{M}$ sucrose, $1 \mathrm{mM}$ EDTA, and $10 \mathrm{mM}$ Hepes, $\mathrm{pH}$ 7.0), and then centrifuged at $1500 \mathrm{~g}$ at $4{ }^{\circ} \mathrm{C}$ for $10 \mathrm{~min}$ to remove the nuclei and intact cells. Postnuclear supernatants were then subjected to ultracentrifugation through a Percoll density gradient using an ultracentrifuge. An ultracentrifuge tube was layered with $2.5 \mathrm{M}$ sucrose, $18 \%$ Percoll in HM buffer, and supernatant (top). The centrifugation was performed at $90,000 \mathrm{~g}, 4^{\circ} \mathrm{C}$, for $1 \mathrm{~h}$. Samples were fractionated into light, medium, and heavy membrane fractions. Heavy membrane fractions contained concentrated bands of cellular organelles and were further layered over a discontinuous iodixanol gradient, generated by mixing iodixanol in $\mathrm{HM}$ buffer with $2.5 \mathrm{M}$ glucose (in vol/vol; 27, 22.5, 19, 16, 12, and $8 \%$ ) and with osmolarity maintained at $300 \mathrm{mOsm}$ for all solutions. After centrifugation at $4{ }^{\circ} \mathrm{C}$ for $2.5 \mathrm{~h}$ at $180,000 \mathrm{~g}(44,200 \mathrm{rpm})$, each sample was divided into 12 fractions $(0.5 \mathrm{ml}$ each) and the first two fractions were used for further analyses. Cell surface proteins were isolated as previously [31] by using pierce cell surface protein isolation kit. 
Overall, $10-20 \mu \mathrm{g}$ of total protein was loaded for each sample into 8 or $14 \%$ gels. Gels were transferred onto Nitrocellulose Blotting Membranes (Bio-rad). Antigenspecific primary antibodies were incubated overnight at $4{ }^{\circ} \mathrm{C}$ and detected with species-specific far-red fluorescentlabeled secondary antibodies (Li-Cor). Band quantification was performed using ImageJ software (version 1.44; NIH). Bands of interest were normalized to beta-actin or GAPDH for a loading control.

\section{Cell line and transfection}

Neuro-2a cells (ATCC, RRID: CVCL_0470) were maintained in DMEM medium (Invitrogen) supplemented with $10 \% \mathrm{FBS}$ at $37{ }^{\circ} \mathrm{C}$ and $5 \% \mathrm{CO}$. DNA transfection was performed using a standard polyethyleneimine protocol. They were authenticated based on morphology, and DNA staining revealed no mycoplasma contamination.

\section{Cultured cortical neuron preparation}

Primary dissociated cortical neuronal cultures were prepared from E14.5 mouse embryos, according to protocols previous used for hippocampal neurons [27, 32, 33], with the added step of straining the cells through a Falcon Cell Strainer (Thermo Fisher Scientific Cat\# 08-771-1). Mouse cortical neurons were plated on poly-L-lysinecoated coverslip at 50,000-80,000 cells $/ \mathrm{cm}^{2}$, in Neurobasal medium (Invitrogen, Carlsbad, CA) containing B-27 (Invitrogen), $2 \mathrm{mM}$ Glutamax-I (Invitrogen), and 2.5\% FBS (HyClone, Logan, UT). On the second day in vitro (DIV 2), medium was half changed to medium without FBS. Neurons were fixed with $4 \%$ PFA, $4 \%$ sucrose in PBS for immunocytochemistry at DIV 10-12. Immunostaining of neuronal cultures was performed as described previously $[10,12,14]$.

\section{Quantitative real-time RT-PCR (qRT-PCR)}

Total RNA from both mutant and littermate control mice brain was extracted using TRIzol reagent (Life Technologies) as previously described [34, 35]. After homogenizing the samples with TRIzol reagent, chloroform was added and the aqueous layer containing the RNA was isolated and precipitated with isopropanol. Reverse transcription into cDNA was carried out by using the iScript cDNA Synthesis Kit (Cat. \#: 170-8890, Bio-RAD) according to manufacturers' instructions. qRT-PCR was performed with a Bio-Rad CFX96 Real-Time system using Maxima SYBR Green/ROX qPCR Master Mix (Thermo Fisher Scientific). Samples were run in triplicates and all signals were normalized to GAPDH intensity.

\section{Statistics}

Although no statistical methods were used to predetermine sample size, our sample sizes are based on our previous experiences or publications [36-39]. All data are presented as mean \pm SEM. GraphPad Prism 8 (GraphPad Software) was used for statistical analysis. Data distribution was assumed to be normal, but this was not formally tested. The two-tailed unpaired $t$-test was used to evaluate statistical significance of two groups of samples. One-way analysis of variance (ANOVA) with a Tukey post hoc test was used to evaluate statistical significance of three or more groups of samples. The $n$-numbers can be found in the figure legends. Statistical significance was defined as $P<0.05$.

\section{Results}

\section{Requirement of Vps35 in embryonic pyramidal neurons for axonal and dendritic terminal differentiation}

Studying in vivo function of Vps35 has been difficult because Vps35 null allele die before E9.5 with an unknown reason [10]. To determine Vps35's function during neocortical development, we performed IUE to delete Vps35 in a subset of neocortical pyramidal neurons. Cre-encoding plasmids were electroporated into the neural stem cells (NSCs) or neural progenitor cells (NPCs) in the ventricular zone of $V p s 35^{f / f}$ embryos at E14.5. These NSCs/NPCs differentiate into neocortical pyramidal neurons at layer $(\mathrm{L})$ 2-3 at neonatal age (Fig. 1a) [40]. As expected, expression of the Cre-GFP abolished endogenous Vps35's expression (Fig. 1d inserts). At P7, most of $\operatorname{Vps} 35^{K O}\left(\mathrm{Cre}_{\mathrm{GFP}}{ }^{+}\right)$ neurons had migrated to neocortical L2-3, as that of control $\left(\mathrm{GFP}^{+}\right)$neurons (Fig. 1b). Their dendritic morphology (Fig. 1d), length (Fig. 1f), complexity (Fig. 1g), and their axonal length and distribution in corpus callosum (CC) (Fig. 1n) appeared to be comparable with those in control neurons. These results suggest little impact if there is any of $V p s 35^{K O}$ on neuron migration and axonal initiation.

However, starting from P14, obvious morphological changes in both dendrites and axons were found in $\mathrm{Vps} 35^{\mathrm{KO}}$ neurons (Fig. 1e). Quantification of the total dendritic length indicated a progressive reduction of dendritic processes in $V p s 35^{K O}$ neurons (Fig. 1f). Sholl analysis, which quantifies the complexity of the dendritic arbor, showed much lower number of dendritic intersections in $\operatorname{Vps} 35^{K O}$ neurons (Fig. 1h). These results suggest that $\operatorname{Vps} 35^{K O}$ prevents dendritic arborization during neuronal maturation. Dendritic arborization and spine formation are closely related, we then examined spine morphology and quantified spine density. 

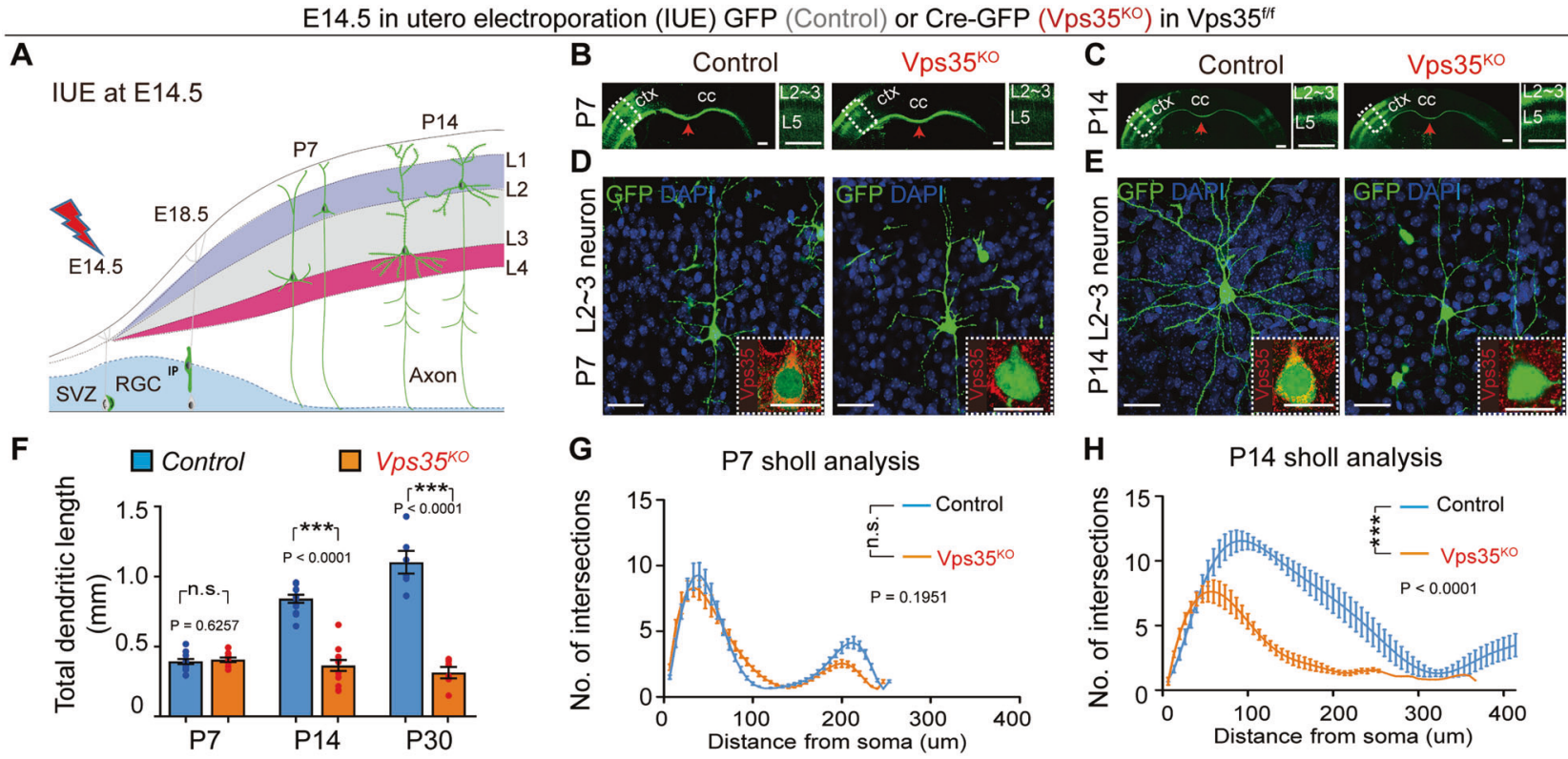

I
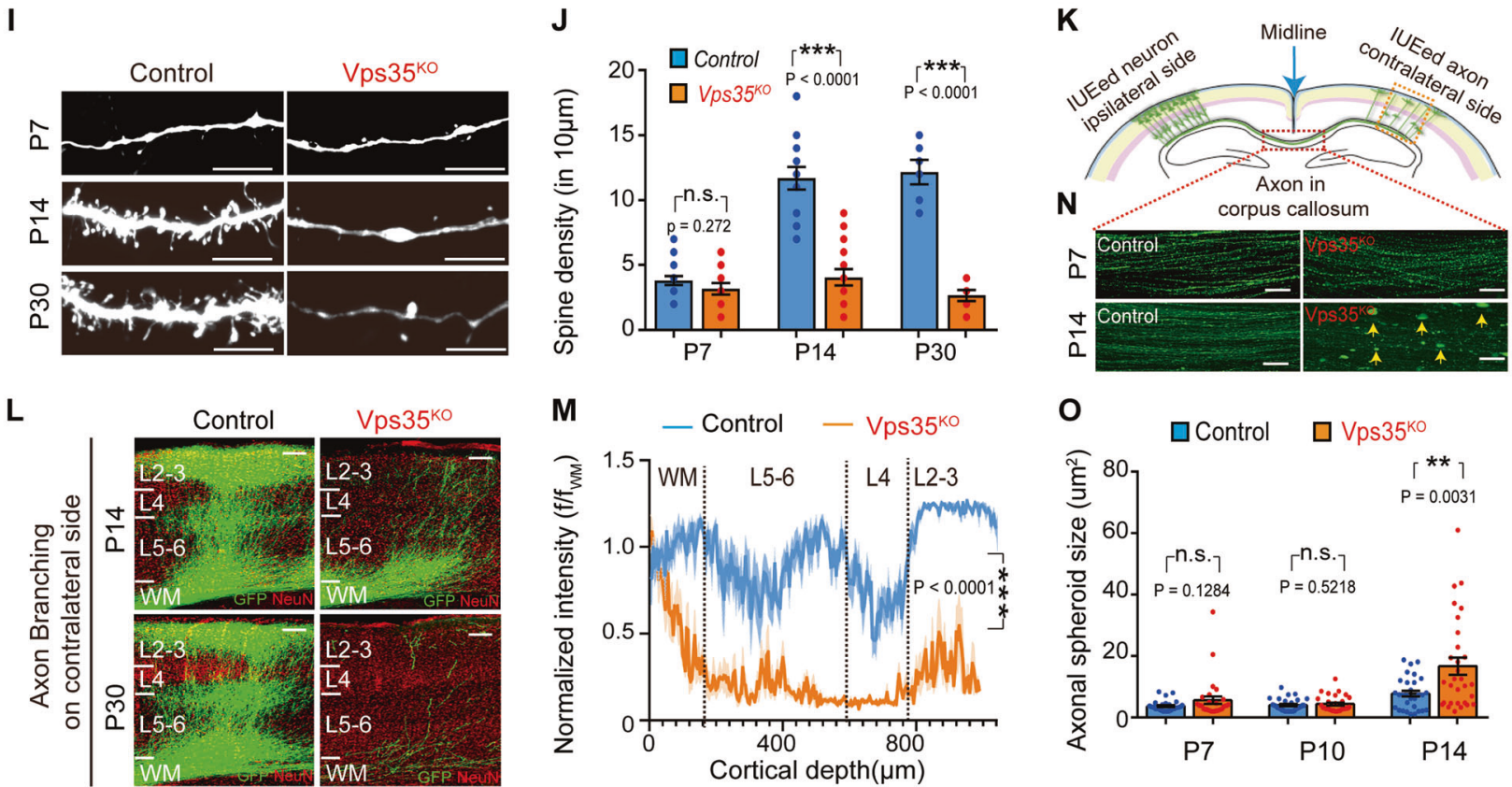

Fig. 1 Defective dendritic and axonal morphogenesis in neonatal pyramidal neurons by depletion of Vps35 at E14.5 NSCs/NPCs. $\mathrm{Vps}^{\mathrm{f}} 5^{\mathrm{f} / \mathrm{f}}$ embryos were in utero electroporated (IUEed) with plasmids of GFP (control) or Cre-GFP (Vps35 ${ }^{\mathrm{KO}}$ ) at E14.5 and their neocortical brain sections were examined at indicated ages. a Schematics of E14.5 IUE experimental procedures. b, c Representative images from neocortical brain sections at indicated ages. Cerebral cortex (ctx), corpus callosum (cc), and middle lines (red arrows) are marked. Images in dashed lines are amplified in the right panels. Cortical layers (L) 2-3 or 5 are indicated. d, e Representative Z-stack projection images of $\mathrm{GFP}^{+}$ (electroporated) L2-3 cortical neurons. The inserts are representative images of immunostaining analysis with anti-Vps35 (red). f Quantification analysis of the cumulative length of the dendritic processes of L2-3 neurons. ( $n=12$ neurons from four mice per group, unpaired two-tailed $t$-test). $\mathbf{g}, \mathbf{h}$ Sholl analyses of dendritic intersections from neuronal soma. ( $n=4$ neurons from three mice per group; two-way ANOVA with a Tukey's multiple comparisons test). i Examples of reconstructed dendritic branch segments and their associated spines at indicated ages. j Quantification analysis of the spine density. ( $n=16$ neurons from four mice per group, unpaired two-tailed $t$-test). $\mathbf{k}$ Diagram showing IUEed axons in corpus callosum (CC) crossing the midline and then innervation into contralateral cortex and branching extensively at in layers 2-3 and layer 5. I Higher magnification of contralateral side showing reduced axon innervation and branching in Vps35-KO neurons. m Quantification of fluorescence intensity across layers normalized by fluorescence of the underlying white matter $\left(\mathrm{f} / \mathrm{f}_{\mathrm{wm}}\right)$ from P14 sections. n Representative images of $\mathrm{GFP}^{+}$axon taken from the illustrated area in $\mathbf{k}$. The yellow arrows indicate the axonal spheroids. o Quantification analysis of data in $\mathbf{n}$. $(n=30$ from six mice per group, unpaired two-tailed $t$-test). Scale bars: in $\mathbf{b}, \mathbf{c}$ and $\mathbf{l}$, $500 \mu \mathrm{m}$; and in $\mathbf{d}, \mathbf{e}, \mathbf{i}$, and $\mathbf{n}, 10 \mu \mathrm{m}$. Individual data points were shown as dots with group mean \pm s.e.m; $* * P<0.01$, $* * * P<0.001 ;$ n.s. not significant. 
By 3D reconstruction, a significant reduction in total spine density was detected in $\operatorname{Vps} 35^{K O}$ neurons at P14 and P30 (Fig. 1i-j).

As IUE was performed unilaterally in the somatosensory cortex, and the axon of electroporated neurons was determined by expression of GFP, which allowed us to check the axonal morphology at high resolution. As shown previously [22], callosal axons cross the cortical midline, forming the $\mathrm{CC}$ around birth, and then reach the contralateral cortex during the first postnatal week and branch extensively at P14 which targeting the contralateral layers 2-3 and layer 5 neurons (Fig. 1k). Vps35 ${ }^{K O}$ axons formed, grew, and successfully crossed the midline (Fig. 1b, c), but failed to branch and innervation contralateral side neurons at P14. These deficits seemed to be more severe at P30 (Fig. 11 and $\mathrm{m})$. In addition, a marked increase of axonal spheroid formation (viewed by GFP with area size $>10 \mu \mathrm{m}^{2}$ ) was observed in $\mathrm{Vps} 35^{K O}$ axons in CC (Fig. 1n and o).

To further determine neuronal Vps35's function, we generated Vps35f/f; Neurod6-Cre (referred to hereafter as

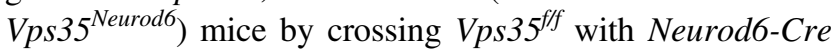
(also called Nex-Cre). The Neurod6-Cre is expressed exclusively in pyramidal neurons beginning at approximately E11 [21]. Vps35 $5^{\text {Neurod6 }}$ mice exhibited no obvious differences from control littermates at birth (Fig. S1A). However, they lagged in postnatal development including cessation of weight gain after 1 week and neonatal lethality before P23 (Fig. S1A, B). Consistent with Neurod6-Cre, Vps35 was depleted in differentiated pyramidal neurons in neocortex by immunostaining analysis (Fig. S1C). Western blot analysis also showed selective reductions of $\mathrm{Vps} 35$ protein levels in Vps35 $35^{\text {Neurod6 }}$ neocortex and hippocampus $(59.3 \pm 6.29 \%$ reduction in neocortex and $56.61 \pm 3.19 \%$ in hippocampus), but not in other brain regions where the Cre is not expressed (Fig. S1D).

To better view the morphology of individual pyramidal neurons in Vps35 $35^{\text {Neurod6 }}$ mice, we crossed Vps $35^{\text {Neurod6 }}$ with a Thyl-EYFP mice, which fluorescently labels a proportion of neurons in neocortex. The EYFP expressing dendrites from Vps35 $5^{\text {Neurod6 }}$ mice was comparable with control mice at P7 (Fig. 2a-c). However, at P14, these Vps35 Neurod6 neurons showed similar deficits as that detected by IUE assay, which include reduced dendritic length (Fig. 2d, e) and complexity (Fig. 2f), decreased dendritic spine density (Fig. 2g, h), and impaired mature spine formation (Fig. 2i, j). Moreover, Vps35 $5^{\text {Neurod6 }}$ axons in $\mathrm{CC}$ also displayed increased axonal spheroids (Fig. $2 \mathrm{k}-\mathrm{l}$ ). The dendritic phenotypes were further confirmed by Golgi staining analysis of P14 control and Vps35 $5^{\text {Neurod6 }}$ cortical brains. Not only L5 neurons, nearly all neurons marked by Golgi staining showed abnormal morphology in the mutant neocortex, including reduced dendritic length and complexity and decreased dendritic spine density (Fig. S2). These results suggest a function of $\mathrm{Vps} 35$ in promoting dendritic and axonal maturation in developing pyramidal neurons.

\section{Concurrence of neurodegenerative and reactive glial responses in Vps35 $5^{\text {Neurod6 }}$ neocortex}

The morphological changes in neonatal Vps35 $5^{\text {Neurod6 }}$ neurons resemble in certain degree to the neurodegenerative pathology for aged neurons [41]. We thus examined if Vps35 $5^{\text {Neurod6 }}$ neocortex shows any feature of neurodegeneration. By analysis of Nissl-stained brain sections, we did not observe any change at P0 and P7 but noticed an obvious reduction in cortical thickness in Vps35 $35^{\text {Neurod6 }}$ at $\mathrm{P} 14$ that was more severe at P21 (Fig. 3a and c). NeuN (a marker for neurons) immunostaining analysis showed a comparable number of cortical neurons at P7 with those of control mice, but losses of $12 \%$ and $29 \%$ of cortical neurons at P14 and P21, respectively (Fig. 3d). Such an age-dependent neuron loss was accompanied by an increase in active caspase- 3 positive apoptotic cells in Vps35 $5^{\text {Neurod6 }}$ neocortex (Fig. 3b and e). In line with the enhanced active caspase-3 staining, the TUNEL assay confirmed an increase of apoptotic cells in P21 Vps35 $5^{\text {Neurod6 }}$ neocortex (Fig. S3A, B). As apoptosis can be induced by various cellular stresses, including DNA damage, the P21 control and Vps $35^{\text {Neurod } 6}$ brain sections were immune-stained with antibodies against $\gamma \mathrm{H} 2 \mathrm{AX}$ and P53, both DNA damage markers [42, 43]. Indeed, both $\gamma \mathrm{H} 2 \mathrm{AX}$ and P53 were increased in Vps35 ${ }^{\text {Neurod6 }}$ neocortex as compared with that of littermate controls (Figs. S3B-C). These results suggest that the DNA damage associated apoptosis may contribute to the progressive cortical neuron loss in Vps35 $35^{\text {Neurod6 }}$ mice.

Notice that the percentage apoptotic cells (less than 1\%) in Vps35 $35^{\text {Neurod6 }}$ neocortex were smaller than that of neuron loss, suggesting additional mechanism(s) underlying cortical brain atrophy. We thus further examined neurodegenerative pathology in Vps35 $5^{\text {Neurod6 }}$ brain sections by staining with NeuroSilver (Fig. 3f) or Jade C (Fig. 3g), or immunostaining using anti-Rtn3 (Fig. 3h), as these three markers are frequently used for detection of neuronal damage or dystrophic neuritis [28, 29, 44]. Indeed, positive signals with NeuroSilver, Jade C, and Rtn3 were all detected in Vps35 $35^{\text {Neurod6}}$, but not control brains (Fig. 3f-k). Interestingly, staining signals of the three markers were stronger in the mutant L2-3 and $\mathrm{CC}$ axon regions (Fig. 3f-k), suggesting a regional difference/selectivity of neurodegeneration. Notice that in the mutant stratum, where the Neurod6Cre is not expressed, the staining signals appeared to be comparable with that in controls (Fig. $3 \mathrm{f}-\mathrm{k}$ ), demonstrating the specificity of the staining.

Neurodegeneration is often associated with glial activation. As shown in Fig. 4a, whereas aldolase $\mathrm{C}$ positive $\left(^{+}\right)$astrocytes in P14 Vps35 $5^{\text {Neurod6 }}$ neonatal cortex was comparable 
A

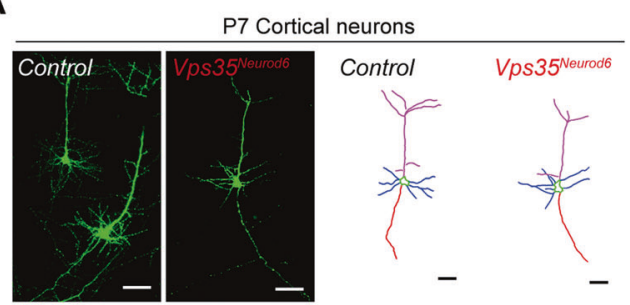

D

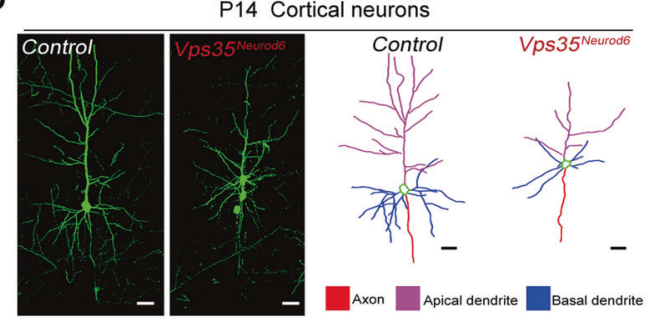

G

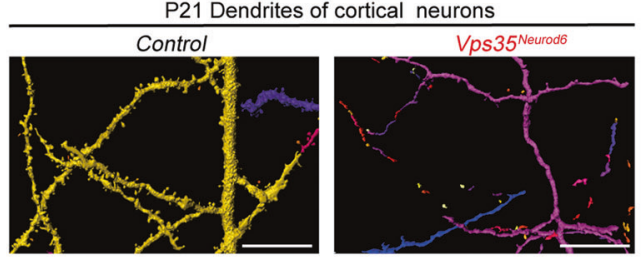

I

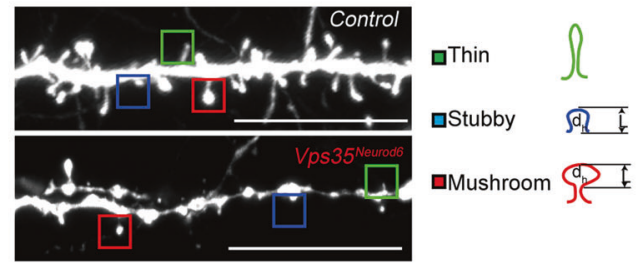

B

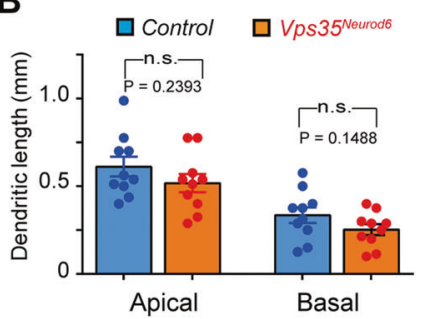

E

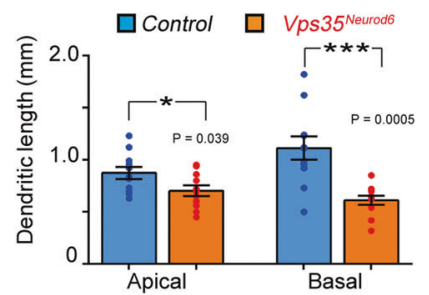

H

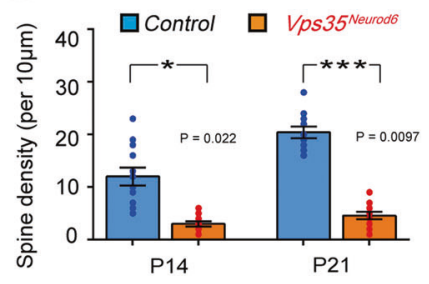

J

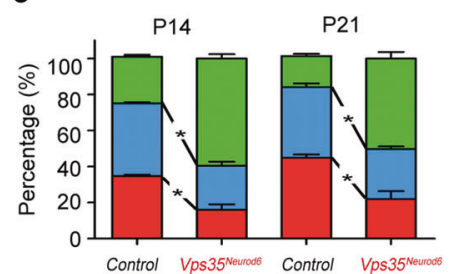

C

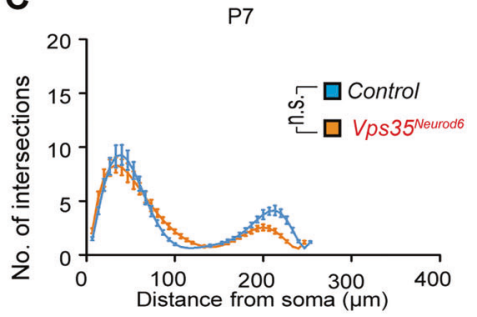

$\mathbf{F}$

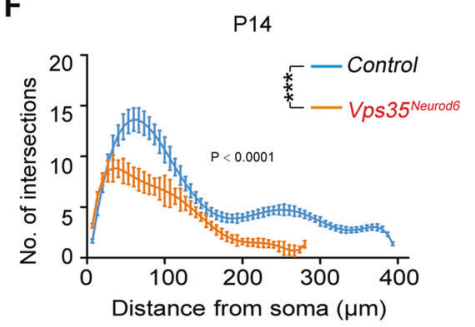

K

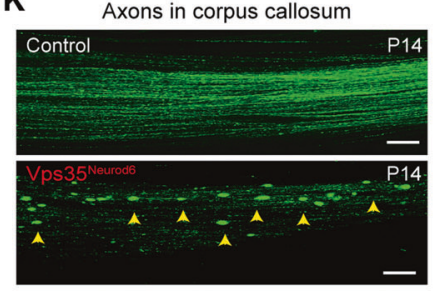

$\mathbf{L}$

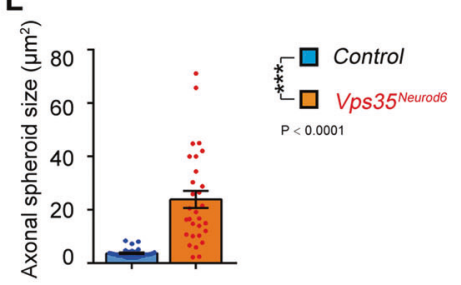

Fig. 2 Dendritic maturation defect and axonal spheroid formation in Vps35 ${ }^{\text {Neurod6 }}$ neocortex. a, d Representative images of EYFP ${ }^{+}$ cortical neurons (left) and reconstructed neurons (right) at P7 and P14. b, e Quantification analysis of the apical and basal dendritic length. ( $n=12$ neurons from four animals per genotype; unpaired two-tailed $t$-test). c, f Sholl analysis for dendritic complexity. (two-way ANOVA with a Tukey's multiple comparisons test). g Representative 3Dreconstructed dendritic spines from P21 brain sections. h Quantifications of spine density at ages of P14 and P21. ( $n=12$ from four mice per group, unpaired two-tailed $t$-test). i Representative Z-stack

with those of control brains, $\mathrm{Gfap}^{+}$astrocytes were significantly elevated in the mutant neocortex, suggesting an increase of reactive astrogliosis, but not astrogliogenesis or astrocyte differentiation (Fig. 4a, b). In accord, $\mathrm{Iba}^{+}$as well as $\mathrm{Cd} 11 \mathrm{~b}^{+}$microglia were both increased in the mutant neocortex (Fig. 4c, d), indicating an elevation of microglial activation. This view was in line with the observation of altered $\mathrm{Iba}^{+}$microglial morphology in Vps $35^{\text {Neurod } 6}$ neocortex, which had larger cell body and shorter filopodium-like processes than those in the controls (Fig. 4e, f), exhibiting morphological features of microglial activation. The increased projection images of dendritic spines. Spines are categorized as mushroom (red), stubby (blue), and thin (green) subtypes. j Quantification analysis of data in $\mathbf{h}$ for percentage of different types of spines of neurons at ages of P14 and P21. (Four neurons from four mice per group, two-way ANOVA with Tukey's multiple comparison test). $\mathbf{k}$ Confocal images of $\mathrm{GFP}^{+}$projecting axons in CC after IUE of CAGGFP at E14.5 control (Vps35 $\left.5^{\mathrm{f} / \mathrm{f}}\right)$ and Vps35 $5^{\text {Neurod6 }}$ embryos. I Quantification analysis of data in $\mathbf{k}$. $(n=30$ from three mice per group, unpaired two-tailed $t$-test). Individual data points were shown as dots with group mean \pm s.e.m. $* P<0.05$; $* * * P<0.001$; n.s. not significant.

Gfap and Iba1 protein levels in Vps35 $5^{\text {Neurod6 }}$ cortical homogenates (from P14, but not P0 and P7, brains) was also detected by western blot analyses (Fig. $4 \mathrm{~g}, \mathrm{~h}$ ), providing additional support for elevated reactive astrogliosis and microglial activation.

\section{Increased autophagosome and neurodegeneration associated proteins in Vps $35^{\text {Neurod6 }}$ brains}

To further address whether Vps35 $5^{\text {Neurod6 }}$ neocortex show neurodegeneration-like pathology, we specifically examined 
A

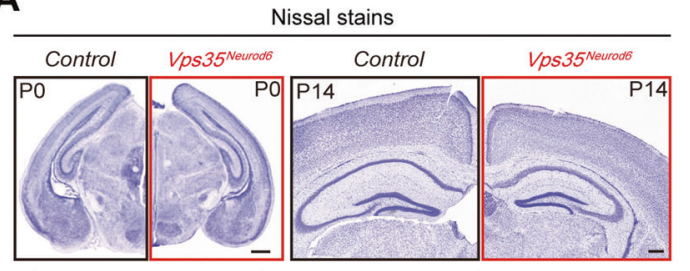

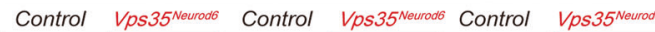

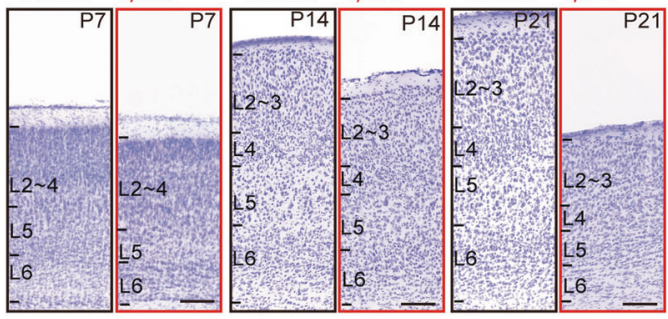

C

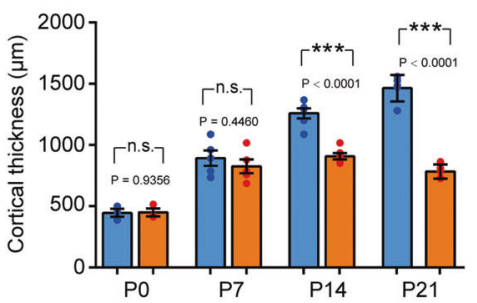

F

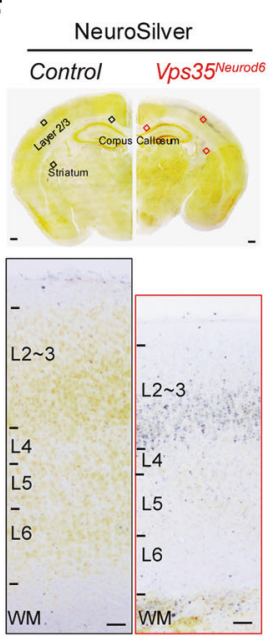

I

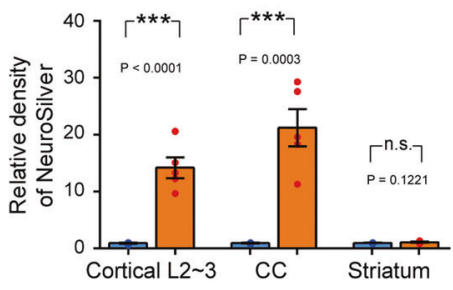

D

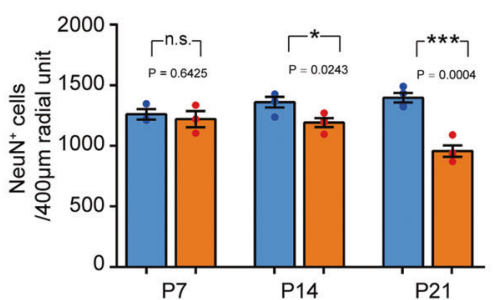

Active caspase-3

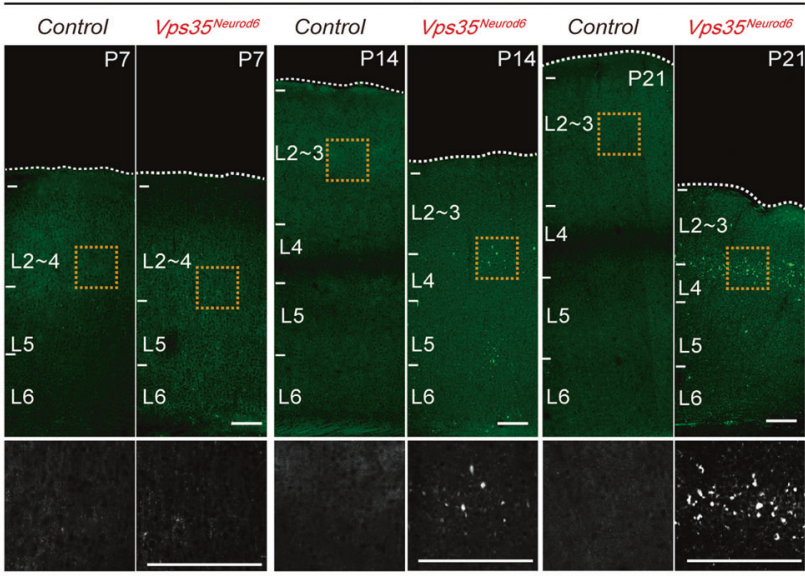

E

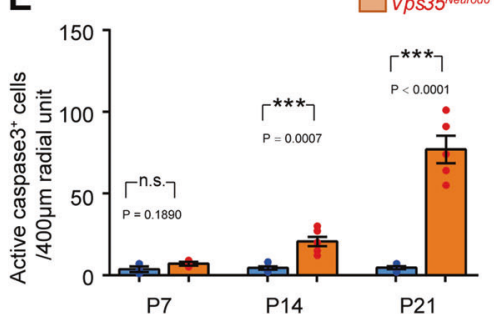

H
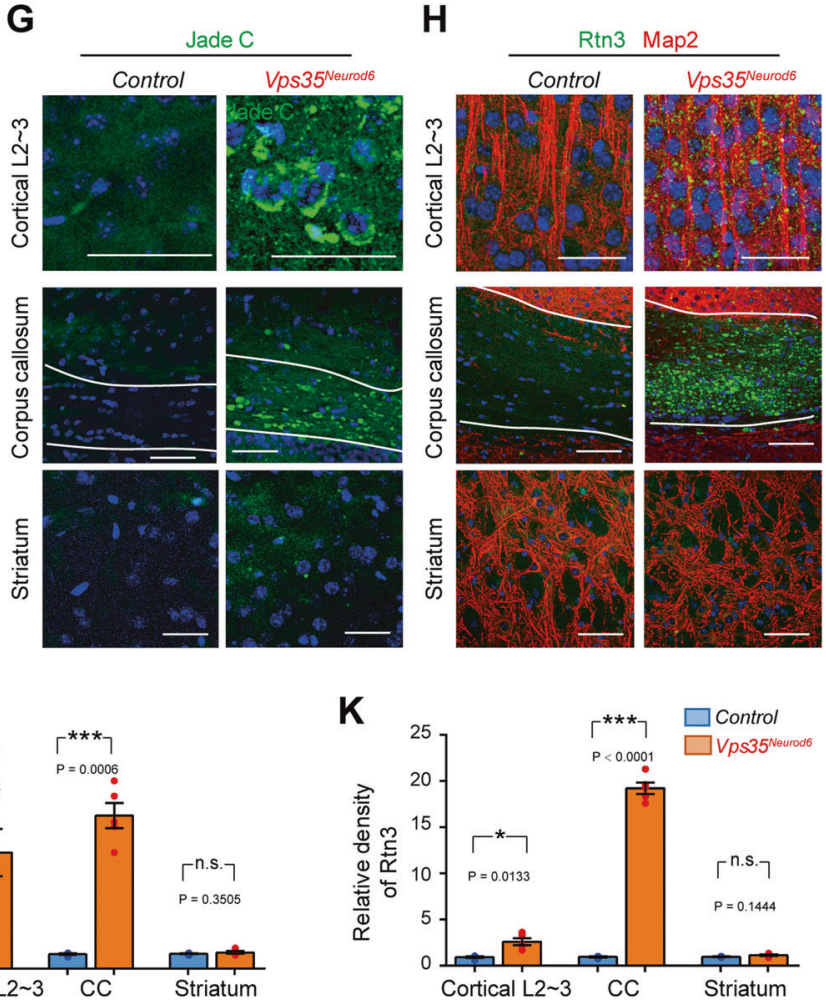

FTD associated proteins, as FTD is the 2nd most common younger-onset dementia that is due in large to the neurodegeneration in pyramidal neurons of frontal lobe [45]. In most of FTD cases, neocortical pyramidal neurons contained pathological protein aggregation of TDP43 and P62 [46]. Western blot analysis showed increased P62 and 
Fig. 3 Degenerative cortical neurons in Vps35 $5^{\text {Neurod6 }}$ brain. a

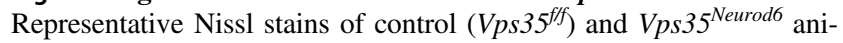
mals at indicated ages. b Representative images of active caspase-3 immunostaining analysis of neocortex at indicated ages. c Quantification analysis of Nissl stains that revealed a significant reduction in cortical thickness of Vps35 $35^{\text {Neurod } 6}$ animals at P14/P21, but not at P0/P7, as compared with those of control mice ( $n=3-4$ animals per genotype; two-tailed unpaired $t$-test). d Quantification analysis of $\mathrm{NeuN}^{+}$ neuron numbers in the neocortex that revealed no significant change at P7, but losses of $12 \%$ and $29 \%$ of cortical neurons at P14 and P21, respectively ( $n=3-4$ animals per genotype; two-tailed unpaired $t$ test). e Quantification analysis of active caspase- $3^{+}$cells that showed an age-dependent increase in apoptotic cells in Vps $35^{\text {Neurod6 }}$ neocortex at indicated ages ( $n=3-4$ animals per genotype; two-tailed unpaired $t$ test). f Images of NeuroSilver-stained brain sections from control and Vps35 $35^{\text {Neurod } 6}$ mice. Positive signals (dark gray/black staining) were detected in cortical L2-3 neurons and corpus callosum [major white matter (WM) axonal pathways], but not in striatum. Representative images of Jade C staining (g) and anti-Rtn3 immunostaining (h) in cortical L2-3, corpus callosum, and striatum of Control and Vps $35^{\text {Neurod } 6}$ mice. i-k Quantification analysis of data from $(\mathbf{f}-\mathbf{h}) .(n=$ 4 animals per genotype; two-tailed unpaired $t$-test). Scale bars: in $\mathbf{a}, \mathbf{b}$, and f, $100 \mu \mathrm{m}$; and in $\mathbf{g}$ and $\mathbf{h}, 20 \mu \mathrm{m}$. Individual data points were shown as dots with group mean \pm s.e.m; $* P<0.05$; $* * * P<0.001$; n.s. not significant.

Tdp43 levels in insoluble fractions of homogenates of P14 Vps35 $5^{\text {Neurod6 }}$ cortex (Fig. 5a, b). In addition, a 20-25 kDa band (likely to be a cleaved C-terminal fragment of $T d p 43$ ) was detected in Vps35 $5^{\text {Neurod6 }}$ insoluble fractions (Fig. 5a, asterisk). Immunostaining analysis showed a more dramatic increase of P62 in the neocortex of P14 Vps35 $5^{\text {Neurod6 }}$ cortex (Fig. 5c, d). Intracellular aggregates of phosphorylated Tdp43 was also noted in Vps35 $5^{\text {Neurod6 }}$ cortical neurons (Fig. 5d). Furthermore, both ubiquitin-conjugated proteins (viewed by anti-ubiquitin) and LC3-II (a marker of autophagosomes) were markedly increased in the insoluble fractions of homogenates from Vps35 Neurod6 neocortex (Fig. 5e, f). These results provide additional support for Vps35-KO pyramidal neurons to undergo neurodegeneration with characteristics of FTD.

We further analyzed Vps35's mRNAs in control and different groups of human patients with FTD-TDP. Interestingly, Vps35's expression was selectively reduced in the frontal lobe of patients with FTD-TDP carrying Progranulin $(P G R N)$ mutations, but not in FTD-TDP patients without $P G R N$ mutation, as compared with unaffected controls (Fig. 5g). These results support the view for Vps35-deficiency as a potential risk factor for FTD pathogenesis.

\section{Increase of Sort1 in lysosomes of Vps35-KO neurons}

The selective reduction of Vps35's mRNA in the frontal lobe of FTD patients with $P G R N$ mutation leads us to wonder if Pgrn is involved in Vps35-KO-induced FTD-like brain deficits. Pgrn-deficiency is believed to be a risk factor for FTD [47], and it is also implicated in enhancing neurite outgrowth and neural survival in primary cultured neurons [48], and in regulating neuronal lysosomal function [49, 50]. Interestingly, the Pgrn levels were reduced in Vps35 $5^{\text {Neurod6 }}$ neocortex (Fig. S4A, B). We thus asked if the decreased Pgrn in Vps $35^{\text {Neurod6 }}$ brain contributes to the Vps35-KO-induced neuropathology. To this end, we generated Pgrn expression plasmid and co-electroporated it with Cre into E14.5 Vps35 f/f embryos, and asked whether Pgrn expression in Vps35-KO neurons could diminish the dendritic and axonal deficits. To our surprise, no significant difference, in viewing dendritic and axonal phenotypes, was detected in $\mathrm{Vps} 35-\mathrm{KO}\left(\mathrm{Cre}-\mathrm{GFP}^{+}\right)$neurons with or without Pgrn expression (Fig. S4C-G). These results suggest that expression of Pgrn in Vps35-KO neurons is insufficient to rescue Vps35-KO-induced neuronal deficits.

We then examined Sort1, as it not only acts as a receptor for Pgrn [19] but also a cargo of Vps35 in Hela cells and COS-7 Cells [51, 52]. Whereas Pgrn was reduced, Sort1 levels were slightly increased in P1 Vps35 $5^{\text {Neurod6 }}$ neocortex (Fig. S5A, B). Sortl's mRNAs were comparable in Vps35 $5^{\text {Neurod6 }}$ neocortex with that of controls (Fig. S5C). We then examined Sort1 subcellular distribution in Vps35-KO neurons. Elevated Sort1 in both $\mathrm{Rab5}^{+}$endosomes and Lamp $1^{+}$late endosomes/early lysosomes were detected in primary cultured Vps35-KO neurons (Fig. S5D-E). Such an altered Sort1 distribution (increase in Lamp $1^{+}$lysosomes, but decrease in $\mathrm{GM}_{130^{+}}$Golgi) was also detected in neocortical neurons of Vps35 $35^{\text {Neurod } 6}$ brain (Fig. S5F, G). Furthermore, western blot analyses of lysates of purified lysosomal and surface fractions showed an increase of lysosomal Sort1, but a decrease of cell surface Sort1 in Vps35 $5^{\text {Neurod6 }}$ mice (Fig. S5H, I), reconfirmed the observation of increased lysosomal Sort1 in the mutant neurons. These results thus support the view for Sort1 as a cargo of Vps35 in developing pyramidal neurons and implicate a delayed or impaired lysosomal degradation of Sort1 in Vps35-KO neurons.

\section{Sort1 contributing in part to Vps35-KO-induced neurodegenerative pathology}

To further investigate Sort1's contribution to Vps35-KOinduced deficits, we examined if the increased Sort1 in Vps35 $35^{\text {Neurod6 }}$ brain is necessary for Vps35-KO-induced neuropathology. The plasmids encoding shRNA-Sort1 (\#2 and \#3) were generated, but the shR-Sort1 (\#3) efficiently suppressed the expression of Sort1 in vitro and in vivo (Fig. S6A, B). The shR-Sort1(\#3) plasmids were coelectroporated with Cre-GFP into Vps35f/f mouse embryos. Interestingly, dendritic deficits (including reduced 
A

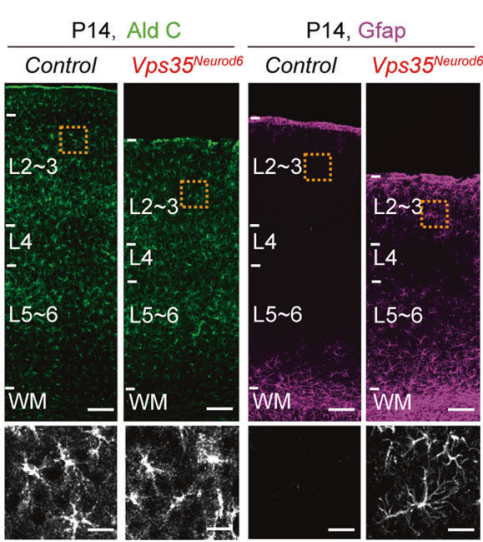

B

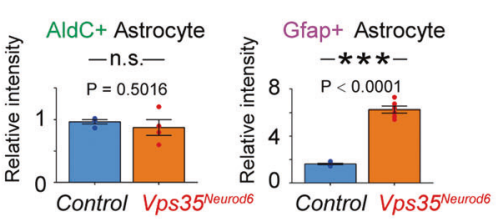

G

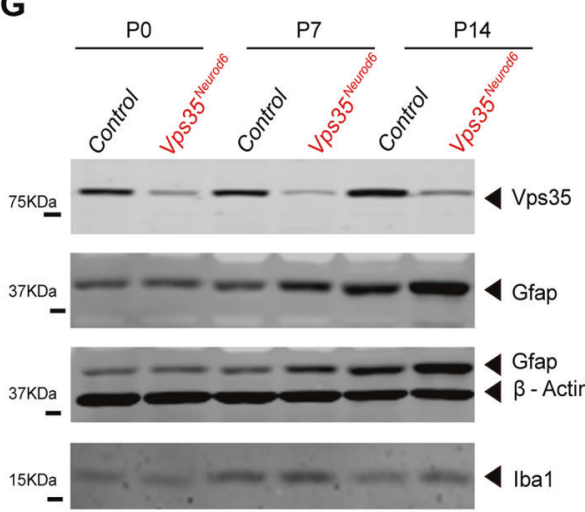

C

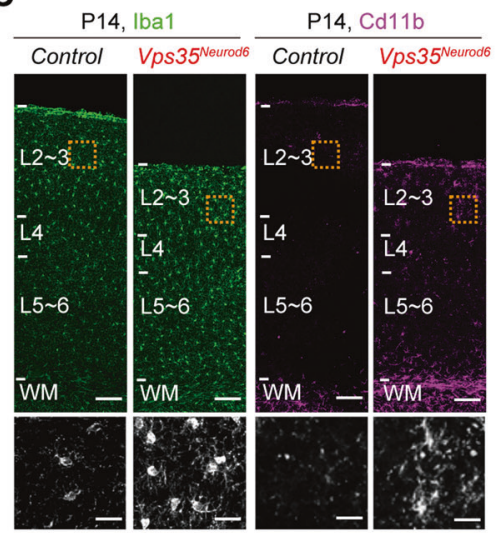

D
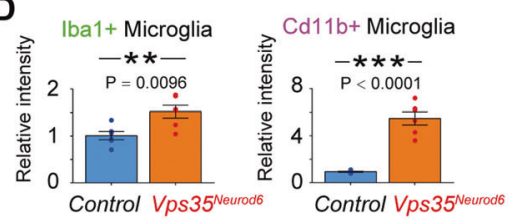

E

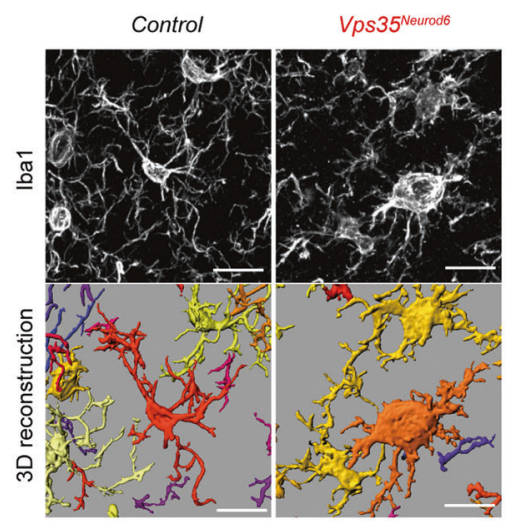

H
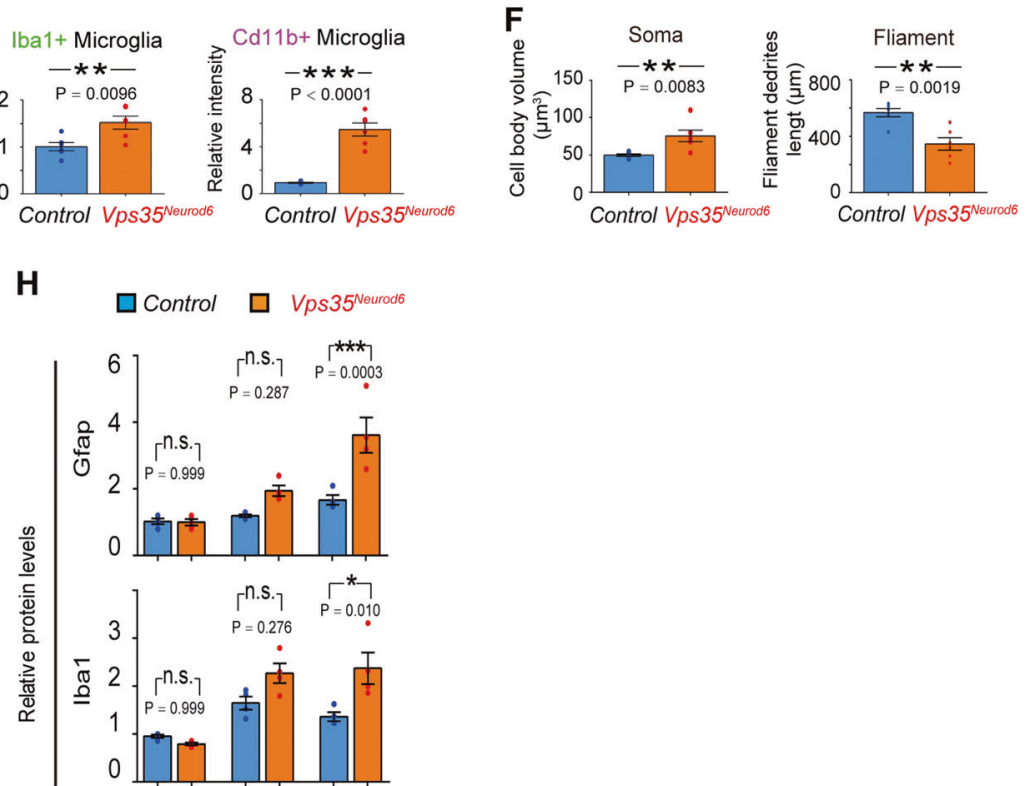

Fig. 4 Reactive astrocytes and microglia in $\mathrm{Vps}_{35} 5^{\mathrm{Neurod} 6}$ neocortex. a, c Immunostaining analysis using indicated antibodies in P14 neocortical sections. Higher-magnification images of the boxed regions were shown in lower panels. b, d Quantification of immunofluorescence from a and c. ( $n=6$ animals per genotype; two-tailed unpaired $t$-test). e, f Representative 3D reconstruction of cortical microglial morphology and Imaris-based automatic quantification of microglial morphology. ( $n=6$ cells from three mice per genotype; two-tailed unpaired $t$-test). $\mathbf{g}, \mathbf{h}$ Western blot analyses and quantification analysis, which confirmed increased Gfap and Iba1 protein levels in the Vps35 $5^{\text {Neurod } 6}$ forebrains at P14, but not $\mathrm{P} 0$ and $\mathrm{P} 7 .(n=4$ animals per genotype; one-way ANOVA with a Tukey's multiple comparisons test). Scale bars: in a, c, and $\mathbf{i}$ (upper panel), $100 \mu \mathrm{m}$; and in $\mathbf{a}, \mathbf{c}, \mathbf{i}$ (lower panel) and $\mathbf{e}, 5 \mu \mathrm{m}$. Individual data points were shown as dots with group mean \pm s.e.m; $* P<0.05 ; * * P<0.01 ; * * * P<0.001$; n.s. not significant. length and complexity and decreased spine density) were diminished when shR-Sortl(\#3) was co-expressed (Fig. 6a-d). However, the axonal spheroid formation remained unchanged (Fig. 6e, f). Notice that the increased P62 in Vps35-KO neurons was attenuated by coelectroporation of shR-Sort1(\#3) (Fig. 6g, h). In addition, the shR-Sortl(\#3) plasmids were electroporated into Vps35 $5^{\text {Neurod6 }}$ embryos (at E14.5), and their P14 dendritic deficits in Vps35 $5^{\text {Neurod } 6}$ neocortex were also diminished by suppression of Sort1 expression (Fig. S6C-G). Together, these results suggest a partial rescue of Vps35-KO-induced neuronal deficit by suppression of Sort1's expression.

\section{Accumulation of lysosomal Sort1 causing similar dendritic and axonal deficits as that of Vps35-KO}

We next wondered if Sort1-increase in lysosomes plays a role in Vps35-KO-induced dendritic and axonal defects. To this end, a plasmid encoding Lyso-Sort 1 fusion protein was generated, in which, Sortl was fused with $C D 63$, a lysosome membrane protein, and thus targeting to lysosomes efficiently and specifically [53] (Fig. 7a, b). Interestingly, when this Lyso-Sort1 fusion plasmid was IUEed into E14.5 embryos, the resulting P14 neocortical neurons exhibited similar deficits as that of Vps35-KO neurons, which include 
A

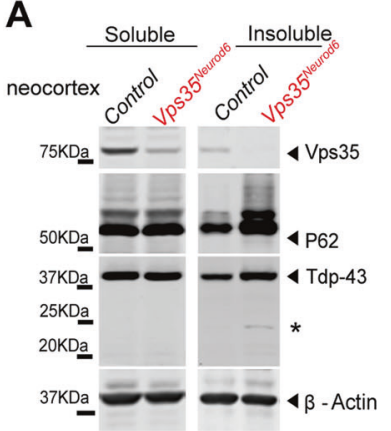

E

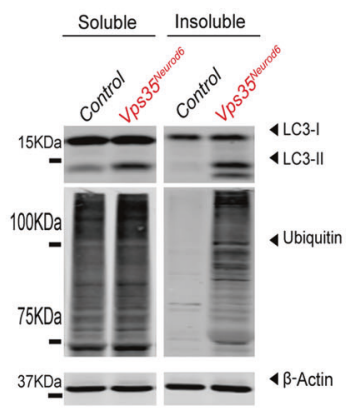

B

Soluble $\square$ Control $\square$ Vps $35^{\text {Nourode }}$ Insoluble Control $\square$ Vps $35^{\text {Nourode }}$

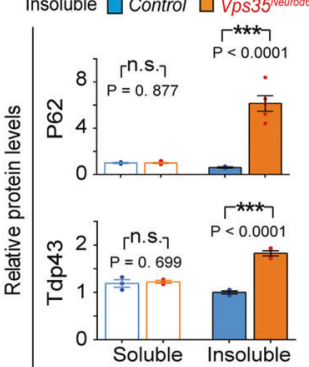

$\mathbf{F}$

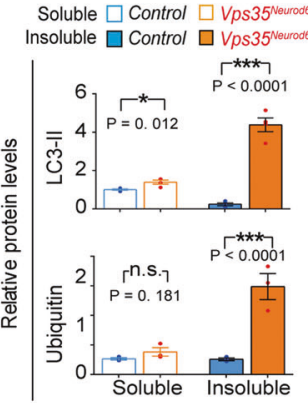

C

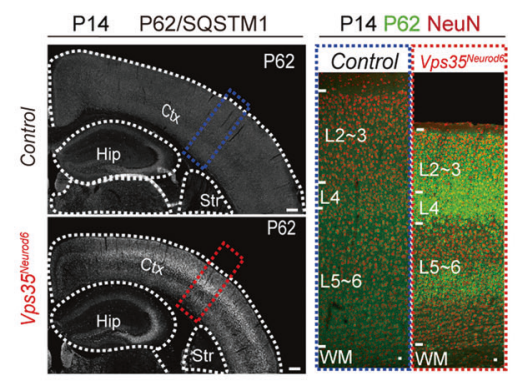

D

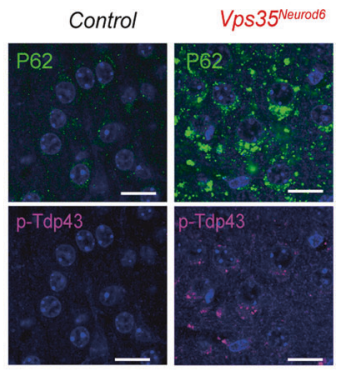

G

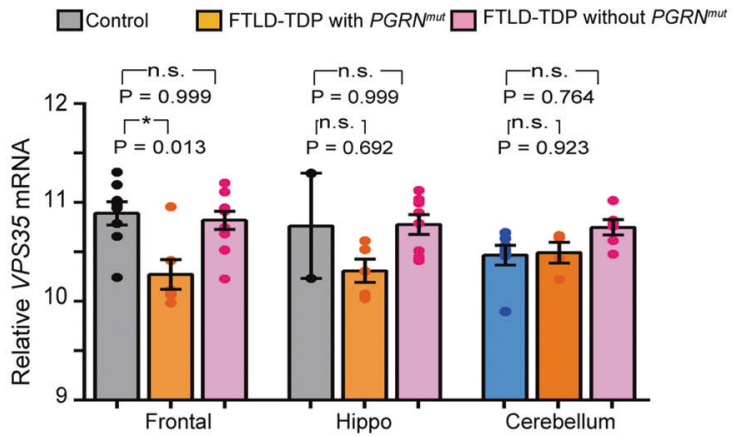

VPS35 mRNA levels in the frontal lobe of cortex (frontal), hippocampus (hippo), and cerebellum from a cohort of eight unaffected (Control), six FTD-TDP patients with Pgrn mutation, and ten FTDTDP without Pgrn mutation. (Data were from GEO GSE20141). Expression levels are normalized to mean of the unaffected group (One-way ANOVA with Tukey's multiple comparison test). Scale bars: in c, $500 \mu \mathrm{m}$; in $\mathbf{d}, 10 \mu \mathrm{m}$. Individual data points were shown as dots with group mean \pm s.e.m; $* P<0.05$, $* * P<0.01$, $* * * P<0.001$; n.s. not significant. impaired dendritic growth, reduced spine density, and axonal spheroid formation (Fig. 7c, d). GFAP ${ }^{+}$astrocytes surround with Lyso-Sortl neurons too (Fig. 7d). In contrast, neurons expressing Lyso-Mock (CD63) appeared to be normal, suggesting the specificity of Lyso-Sortl fusion protein's effect. These results suggest that selective expression of Sort1 in lysosomes is sufficient to recapitulate Vps35-loss-induced terminal differentiation defects.

\section{Lysosomal deficits in both Vps35-KO and Lyso-Sort1 expressing neurons}

The similar deficits between Vps35-KO and Lyso-Sort1 expressing neurons led to the speculation that both Vps35$\mathrm{KO}$ and Lyso-Sort1 expression may cause similar lysosomal defect. Notice that the Lampl $^{+}$lysosomal size was enlarged in both Vps35-KO and Lyso-Sort1 expressing neurons (Fig. 7e), suggesting an alteration of lysosomal morphology. We then examined lysosomal $\mathrm{pH}$ or acidification, an essential event for lysosomal function [54]. The intra-lysosomal $\mathrm{pH}$ was measured by Lyso-pHluorin, which shows strong green fluorescence when it is exposed to a basic or deacidified environment [53] (Fig. 7f). Interestingly, increased Lyso-pHluorin fluorescence intensities were detected in both Vps35-KO and Lyso-Sort1 expression neurons (Fig. 7g), suggesting an impaired lysosomal acidification in both Vps35-KO and Lyso-Sort1 expression neurons. This view was also supported by the observations of increased autophagic protein, $\mathrm{P} 62$, in both Vps35-KO and Lyso-Sort1 expression neurons (Fig. 7h). Together, these results suggest that both Vps35-KO and Lyso-Sort1 may cause dendritic and axonal deficits and neurodegenerative pathology in developing neurons likely by impairing lysosomal acidification.

\section{Discussion}

Vps35 dysfunction is considered to be a risk factor for neurodegenerative diseases, including $\mathrm{AD}$ and $\mathrm{PD}$ [55]. In this paper, we provide evidence that Vps35-deficiency in developing neurons not only impairs axon and dendrite 


\section{A}

Vps35 fff IUE (E14.5 to P14)

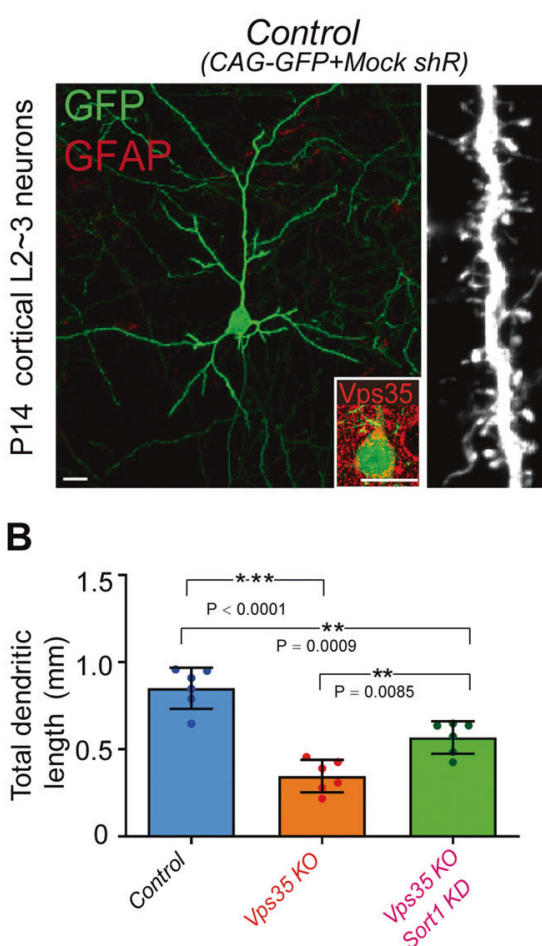

E

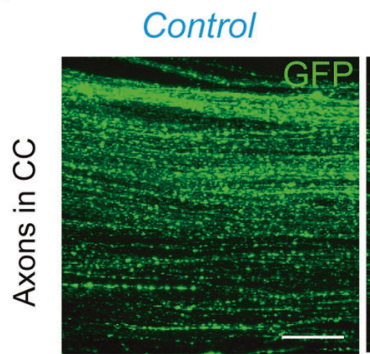

G

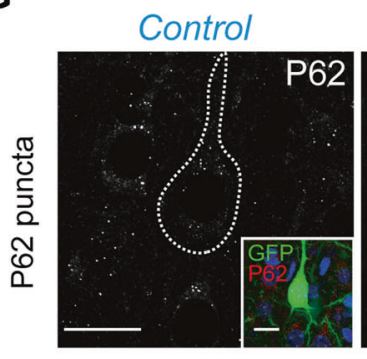

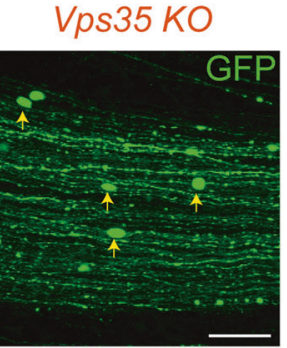

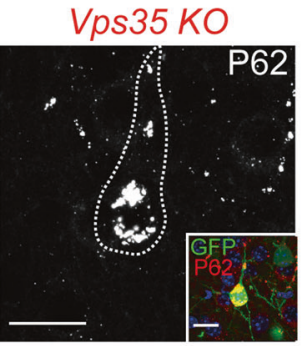

C

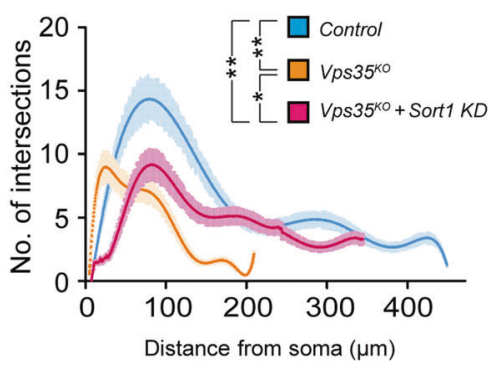

Vps35 KO+Sort1 KD

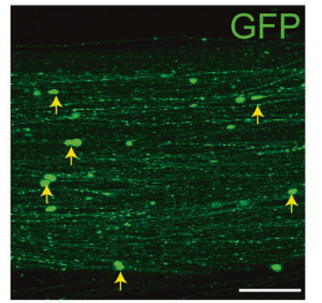

Vps35 KO+Sort1 KD

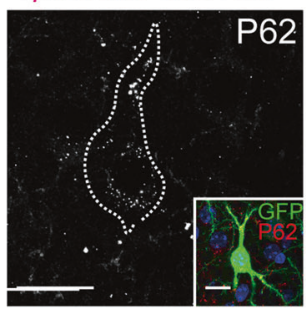

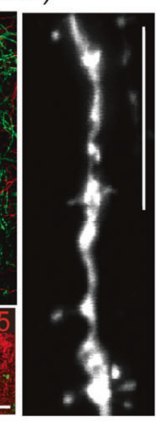

Vps35KO + Sort1KD

(CAG-iCre-GFP+Sort1 shR)

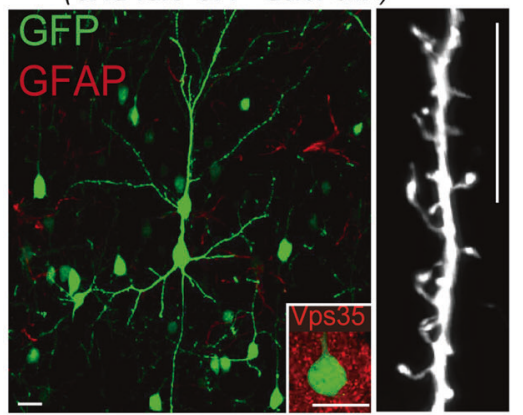

D

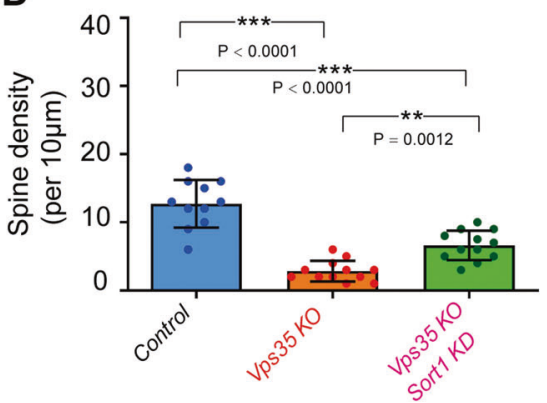

$\mathbf{F}$

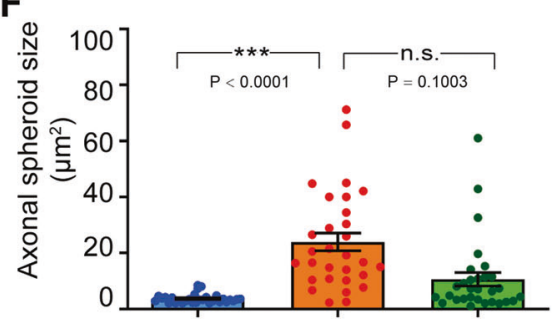

H

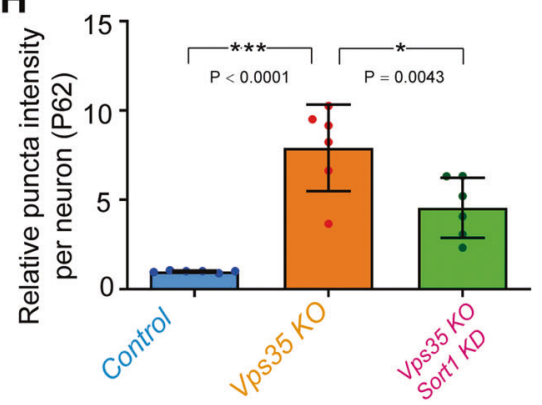

Fig. 6 Attenuated dendritic deficits in Vps35-KO neurons by suppression of Sort1 expression. a Representative images of P14 cortical L2-3 neurons which were electroporated at E14.5 into Vps35/f embryos with the indicated plasmids. The inserts are representative images of immunostaining analysis with anti-Vps35. Quantification analysis of total dendritic length (b), dendritic complexity (c), and spine density (d). $n=6$ neurons from three mice per group; one-way ANOVA with Tukey's multiple comparison test). e Representative images of $\mathrm{GFP}^{+}$projecting axons in CC taken from indicated P14 neocortical brains. $\mathbf{f}$ Quantification analysis of data in $\mathbf{h}(n=30$ from three mice per group, one-way ANOVA with Tukey's multiple comparison test). $\mathrm{g}$ Representative images of immunostaining analysis using anti-P62 of cortical L2-3 neurons from electroporated brain with indicated constructs. Inserts indicated which neurons were transfected. h Quantification analysis of data in $\mathbf{g}$. ( $n=6$ neurons from three mice per group, one-way ANOVA with Tukey's multiple comparison test). Scale bars: in a, e, and $\mathbf{g}, 10 \mu \mathrm{m}$. Individual data points were shown as dots with group mean \pm s.e.m; $* P<0.05, * * P<0.01, * * * P<0.001$; n.s. not significant. 


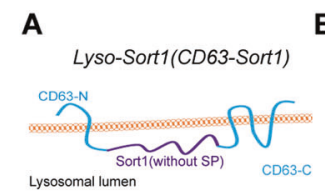

D

B
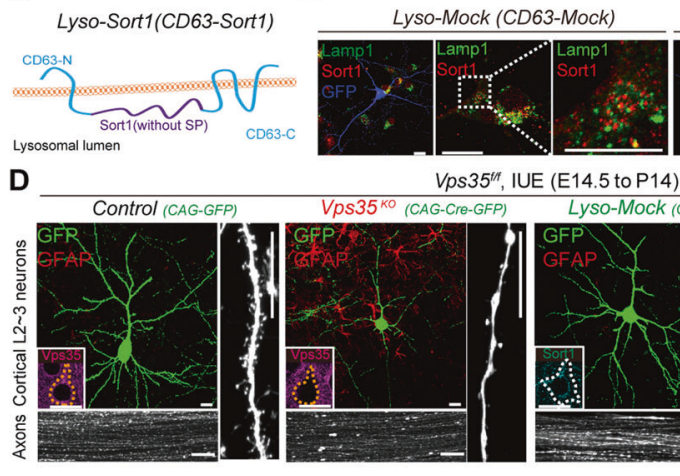

Vps35" ${ }^{\text {fr }}$, IUE (E14.5 to P14)

E

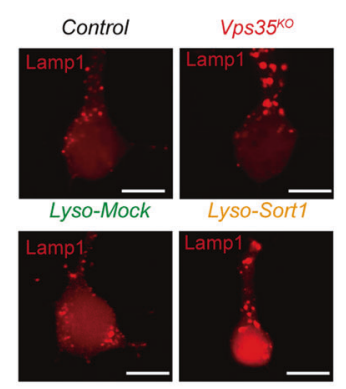

Lamp1 puncta size

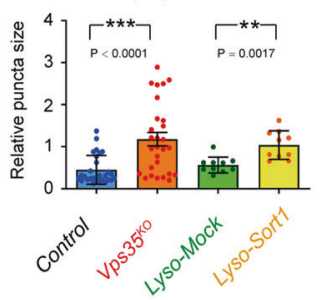

F

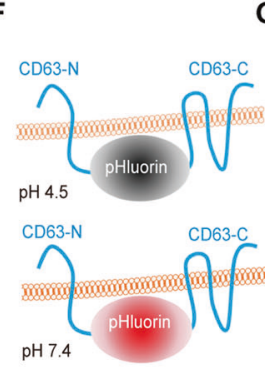

Lyso-Sort1 (CD63-Sort1)

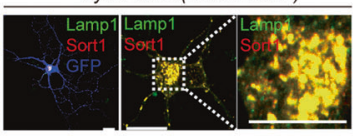

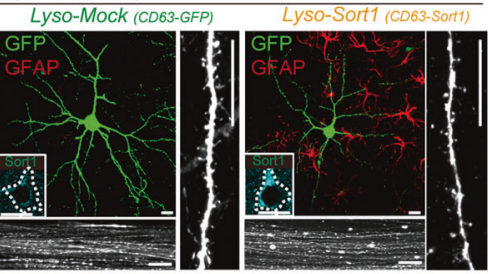

G

Control

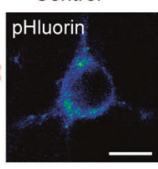

Lyso-Mock

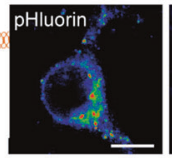

$\operatorname{Vps} 35^{\mathrm{KO}}$

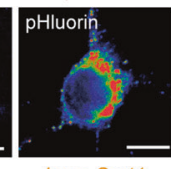

Lyso-Sort1

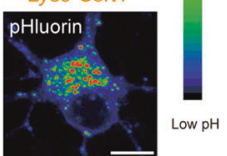

Lyso-pHluorin intensity

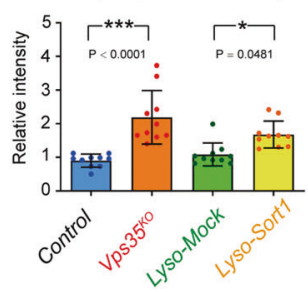

C
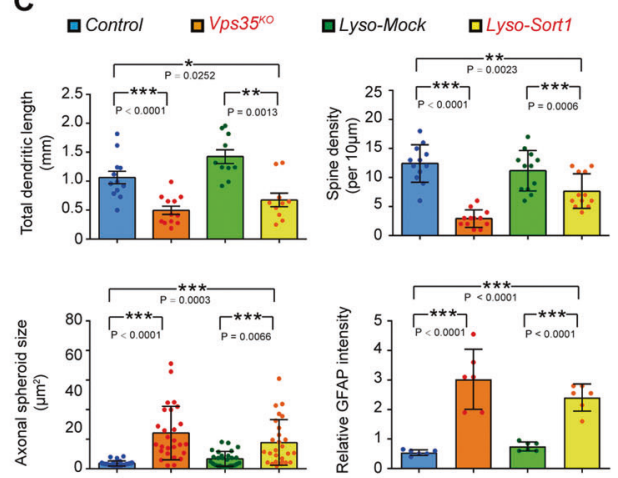

H

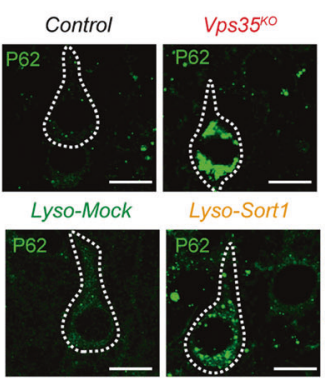

P62 aggregates

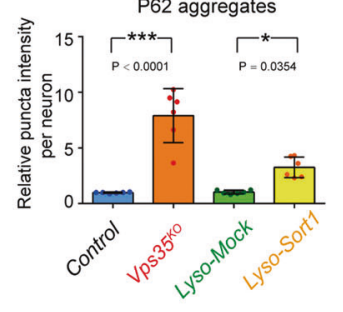

Fig. 7 Impairment in axonal and dendritic terminal differentiation and impaired lysosomal acidification in neurons expressing LysoSort1 fusion protein. a Schematic of the Lyso-Sort1 construct. b Confocal images of a DIV 10 neuron expressing CD63-Mock (LysoMock) and CD63-Sort1 (Lyso-Sort1), which were co-immunostained with anti-Lamp1 (green) and Sort1 (red). c Quantification analysis of total dendritic length, spine density, axonal spheroid size, and relative GFAP intensity from (f). ( $n=12$ neurons from three mice per group; one-way ANOVA with Tukey's multiple comparison test). d Representative images of P14 cortical L2 -3 neurons and their axons in CC which were electroporated at E14.5 with indicated plasmids. The inserts are representative images of immunostaining analysis with antiVps35 (purple) or anti-Sort1 (cyan) in the electroporated neurons. e Representative images and quantitation analysis of Lamp $1^{+}$puncta size in indicated group. Quantification analysis showed increased Lamp1 puncta size in Vps35 $5^{K O}$ and Lyso-Sort1 expressed neurons.
( $n=10$ neurons per group; two-tailed unpaired $t$-test). f A lysosomal target $\mathrm{pH}$-sensitive optical reporter (CD63-pHluorin or Lyso-pHluorin) is quenched when exposed in the acidic environment. When $\mathrm{pH}$ is neutralized, CD63-pHluorin increase the fluorescent intensity. g Representative images of neurons co-transfected with LysopHluorin. Quantification analysis showed increased intra-lysosomal $\mathrm{pH}$ in Vps $35^{K O}$ and Lyso-Sort 1 expressed neurons. ( $n=10$ neurons per group; two-tailed unpaired $t$-test). $\mathbf{h}$ Representative images of immunostaining analysis of P62 in cortical neurons. Quantification analysis showed increased P62 aggregates in Vps35 ${ }^{K O}$ and Lyso-Sort1 expressed neurons and indicative of defects in the clearance of autophagosomes. ( $n=6$ neurons from three mice per group, one-way ANOVA with Tukey's multiple comparison test). Scale bars: in b, d, $\mathbf{e}, \mathbf{g}$, and $\mathbf{h}, 10 \mu \mathrm{m}$. Individual data points were shown as dots with group mean \pm s.e.m; $* P<0.05, * * P<0.01, * * * P<0.001 ;$ n.s. not significant. terminal differentiation, but also causes neurodegenerative pathology. Loss of Vps35 in cortical pyramidal neurons results in dendritic morphogenesis and maturation defects and axonal spheroid formation (Figs. 1 and 2). Vps35-cKO mice, Vps35 $5^{\text {Neurod6}}$, show early onset cortical brain degenerative pathology, including progressive cortical atrophy and increase of neuronal death, "degenerative neuronal morphology" marked by NeuroSilver, Jade C, and Rtn3 (Fig. 3), reactive glial responses labeled by $\mathrm{Gfap}^{+}$ astrocytes and $\mathrm{Cd}_{1} 1 \mathrm{~b}^{+}$microglia (Fig. 4). In addition, Vps35 $35^{\text {Neurod6 }}$ neocortical brains display accumulations of P62, Tdp43, phospho-Tdp43, ubiquitin-conjugated proteins, and LC3-II proteins, exhibiting features of FTD-like neuropathology (Fig. 5). Lysosomes are enlarged with acidification defects in Vps35-KO neurons (Fig. 7). We also uncovered Sort1 as a key cargo of Vps35, whose accumulation in lysosomes contributes to Vps35-KO-induced neuronal deficits (Figs. 6 and 7). Our results not only 
demonstrate a critical role of Vps35 in neuronal terminal differentiation, but also uncover a link of defective terminal differentiation of neurons with neurodegeneration, reveal the importance of lysosomal function in both processes, and implicate Vps35-deficiency as a potential risk factor for FTD development. These findings add further insights into Vps35's function and its involvement in neurodegenerative diseases.

Lysosome and autophagy dysfunctions are known to be key players in the pathogenesis of neurodegenerative diseases $[56,57]$. Here, we speculate that normal lysosomal function may also be required for terminal axonal and dendritic differentiation in developing neurons, because lysosomal dysfunction induced by Vps35-loss or expression of Lyso-Sort1 fusion results in similar deficits in axonal and dendritic terminal differentiation, in addition to neurodegenerative pathology (see Fig. 7). How does Vps35 regulate lysosomal and autophagy functions? Vps35-deficiency in Hela cells reduces proper delivery of proteases to the lysosomes through CI-M6PR; [58, 59] Vps35-deficiency also impairs autophagic degradation in Drosophila [60] and autophagosome formation in Hela cells (through Atg9a); [61] and Vps35-loss increases the degradation of Lamp2a in mouse dopaminergic neurons leading to a decreased chaperone-mediated autophagy [14]. Here, we found that Vps35 regulates lysosomal acidification/maturation in developing pyramidal neurons. While Lamp $1^{+}$early lysosomes are enlarged in Vps35-KO neurons, the acidic lysosomes (or mature lysosomes) are reduced (Fig. 7e-g). In line with this view are our observations of increased P62 and LC3-II autophagic proteins in Vps35 $5^{\text {Neurod6 }}$ pyramidal neurons (Figs. 5 and 7h). Based on our results, we speculate that Sort1, a cargo of Vps35, may be also involved in Vps35 regulation of lysosomal acidification/functions. Intestinally, Sort1 is identified as a binding partner of ATP6AP2, a critical protein for v-ATPase and lysosomal acidification, by proteomics-based approach [62]. Thus, we posit that Vps35-KO in developing pyramidal neurons increases lysosomal Sort1, which may inhibit ATP6AP2 regulated vATPase activity and lysosomal acidification. Whereas these observations support the view depicted in Fig. S7, we cannot exclude the possible involvement of other retromer cargos, such as CI-M6PR [58, 59] and Lamp2a [14], which may also underlie Vps35 regulation of lysosomal functions.

Besides lysosome and autophagy dysfunctions, deregulation of apoptosis is associated with neurodegenerative disorders [63, 64]. Here we showed that ablation of Vps35 in developing pyramidal neurons resulted in not only accumulations of $\mathrm{LC}^{+}$and $\mathrm{P}_{6} 2^{+}$autophagosome markers and FTD-like neurodegenerative pathology, but also an increase of caspase-3-induced apoptosis. Interestingly, both apoptotic neurons [65] and $\mathrm{P}^{2} 2^{+}$inclusions [66] are also found in the cortex of FTD patients. While we believe that the dysfunctional lysosomes contribute to the increase of autophagosome and neurodegeneration associated proteins in Vps $35^{\text {Neurod6 }}$ cortex, the mechanisms underlying the apoptosis remain largely unclear. We speculate the following possibilities. First, Vps35-deficiency may induce DNA damage associated apoptosis by impairing mitochondrial fusion and function and increasing reactive oxygen species (ROS). This view is in line with previous publications that Vps35-deficiency in DA neurons causes neuronal loss by impairing mitochondrial fusion and function [12, 13]. and ROS increases DNA damage [67]. In addition to DA neurons, impaired mitochondrial fusion was also detected in Vps35-deficient pyramidal neurons (data not shown). Whereas these observations support the view for a mitochondrial dysfunction to underlie Vps35deficiency induced apoptosis, further experiments are necessary to determine if ROS is increased and contributes to the DNA damage and apoptosis in Vps35 mutant cortex. Second, it is of interest to note a recent study [68] that shows Vps35 to be a novel regulator of antiapoptotic protein Bcl-xL; and Vps35-depleted cells display an enhanced rate of apoptosis by facilitating Bcl-xL's transport to the mitochondrial outer membrane. Thus, further investigating Vps35's function in regulating Bcl-xL trafficking in cortical neurons and Bcl-xL's involvement in the apoptosis induction in Vps35-deficient neurons are necessary. Third, it is noteworthy that Vps35/retromer is implicated in apoptotic cell clearance through sorting of CED-1, a phagocytic receptor for apoptotic cells identified in Caenorhabditis elegans [69]. Thus, it will be of interest to address if loss of Vps35/retromer in neurons increases apoptotic cells due to an impairment in their clearance by microglial cells. Notice that few $(<1 \%)$ active caspase- $3^{+}$cells contained $\mathrm{P}^{+} 2^{+}$ inclusions (see Fig. S3D-F). Similar results were obtained by TUNEL staining with P62 co-immunostaining analysis (see Fig. S3D-F). Nearly none of the $\mathrm{TUNEL}^{+}$cells had $\mathrm{P}_{62}{ }^{+}$inclusions in the mutant cortex; or nearly all of the $\mathrm{P}^{+} 2^{+}$inclusion containing cells were negative for TUNEL (see Fig. S3D-F). These results support the view that autophagy and apoptosis are two different process [70, 71]. As Vps35/retromer regulates endosomal-to-Golgi trafficking of various transmembrane cargo proteins, it is likely to be involved in both autophagy and apoptosis pathways.

How does Vps35 dysfunction contribute to different neurodegenerative disorders? In light of our observations in various Vps35-cKO mouse models, we speculate that "when" and "where" the Vps35 is lost are crucial factors. Vps35-loss in embryonic cortical neurons during development may predispose to later development of FTD/AD, and Vps35-decrease in DA neurons in SNpc may predispose to a later development of PD [12]. Interestingly, a recent report that knocking down Vps35 in adult hippocampal neurons exhibit comparable dendritic spine/synaptic morphology 
with that of controls [72]. However, AD-relevant deficits, including impaired glutamatergic transmission and decreased long term potentiation are observed in these Vps35-deficient neurons [72, 73]. These different results are likely due to Vps35 functioning in an age and space dependent manners. For example, Vps35 may promote AMPA receptor surface targeting in adult hippocampal/ cortical pyramidal neurons [72-74]. But, in embryonic/ young cortical neurons, Vps35 appears to be critical for Sort1 trafficking. In DA neurons, Mul1, Drp1, and Lamp2a appear to be critical cargos of Vps35, contributing to Vps35-loss-induced PD pathogenesis [12-14]. Thus, at different age and in different type of cells, Vps35 regulates different cargos, therefore, involved in the development of different neurodegenerative disorders.

Acknowledgements We thank Lei Zhang (Case Western Reserve University) for technical support on in utero electroporation. We thank members in Xiong and Mei laboratories for helpful discussions and suggestions.

Funding This work was supported by the National Institutes of Health (AG045781 to WCX).

\section{Compliance with ethical standards}

Conflict of interest The authors declare that they have no conflict of interest.

Publisher's note Springer Nature remains neutral with regard to jurisdictional claims in published maps and institutional affiliations.

Open Access This article is licensed under a Creative Commons Attribution 4.0 International License, which permits use, sharing, adaptation, distribution and reproduction in any medium or format, as long as you give appropriate credit to the original author(s) and the source, provide a link to the Creative Commons license, and indicate if changes were made. The images or other third party material in this article are included in the article's Creative Commons license, unless indicated otherwise in a credit line to the material. If material is not included in the article's Creative Commons license and your intended use is not permitted by statutory regulation or exceeds the permitted use, you will need to obtain permission directly from the copyright holder. To view a copy of this license, visit http://creativecommons. org/licenses/by/4.0/.

\section{References}

1. Forman MS, Trojanowski JQ, Lee VM. Neurodegenerative diseases: a decade of discoveries paves the way for therapeutic breakthroughs. Nat Med. 2004;10:1055-63.

2. Van Deerlin VM. The genetics and neuropathology of neurodegenerative disorders: perspectives and implications for research and clinical practice. Acta Neuropathol. 2012;124:297-303.

3. Vilarino-Guell C, Wider C, Ross OA, Dachsel JC, Kachergus JM, Lincoln SJ, et al. VPS35 mutations in Parkinson disease. Am J Hum Genet. 2011;89:162-7.

4. Zimprich A, Benet-Pages A, Struhal W, Graf E, Eck SH, Offman $\mathrm{MN}$, et al. A mutation in VPS35, encoding a subunit of the retromer complex, causes late-onset Parkinson disease. Am J Hum Genet. 2011;89:168-75.

5. Rovelet-Lecrux A, Charbonnier C, Wallon D, Nicolas G, Seaman $\mathrm{MN}$, Pottier C, et al. De novo deleterious genetic variations target a biological network centered on Abeta peptide in early-onset Alzheimer disease. Mol Psychiatry. 2015;20:1046-56.

6. Burd C, Cullen PJ. Retromer: a master conductor of endosome sorting. Cold Spring Harb Perspect Biol. 2014;6:a016774.

7. Seaman MN. The retromer complex-endosomal protein recycling and beyond. J Cell Sci. 2012;125:4693-702.

8. Wang SA, Bellen HJ. The retromer complex in development and disease. Development. 2015;142:2392-6.

9. Small SA, Kent K, Pierce A, Leung C, Kang MS, Okada H, et al. Model-guided microarray implicates the retromer complex in Alzheimer's disease. Ann Neurol. 2005;58:909-19.

10. Wen L, Tang FL, Hong Y, Luo SW, Wang CL, He W, et al. VPS35 haploinsufficiency increases Alzheimer's disease neuropathology. J Cell Biol. 2011;195:765-79.

11. Muhammad A, Flores I, Zhang H, Yu R, Staniszewski A, Planel E, et al. Retromer deficiency observed in Alzheimer's disease causes hippocampal dysfunction, neurodegeneration, and Abeta accumulation. Proc Natl Acad Sci USA. 2008;105:7327-32.

12. Tang FL, Liu W, Hu JX, Erion JR, Ye J, Mei L, et al. VPS35 deficiency or mutation causes dopaminergic neuronal loss by impairing mitochondrial fusion and function. Cell Rep. 2015;12:1631-43.

13. Wang W, Wang X, Fujioka H, Hoppel C, Whone AL, Caldwell MA, et al. Parkinson's disease-associated mutant VPS35 causes mitochondrial dysfunction by recycling DLP1 complexes. Nat Med. 2016;22:54-63.

14. Tang FL, Erion JR, Tian Y, Liu W, Yin DM, Ye J, et al. VPS35 in dopamine neurons is required for endosome-to-golgi retrieval of Lamp2a, a receptor of chaperone-mediated autophagy that is critical for alpha-synuclein degradation and prevention of pathogenesis of Parkinson's disease. J Neurosci. 2015;35:10613-28.

15. Li JG, Chiu J, Pratico D. Full recovery of the Alzheimer's disease phenotype by gain of function of vacuolar protein sorting 35 . Mol Psychiatry. 2019; Epub ahead of print 7 February 2019; https:// doi.org/10.1038/s41380-019-0364-x.

16. Vieira SI, Rebelo S, Esselmann H, Wiltfang J, Lah J, Lane R, et al. Retrieval of the Alzheimer's amyloid precursor protein from the endosome to the TGN is S655 phosphorylation statedependent and retromer-mediated. Mol Neurodegener. 2010;5:40.

17. Yin J, Liu X, He Q, Zhou L, Yuan Z, Zhao S. Vps35-dependent recycling of Trem2 regulates microglial function. Traffic. 2016;17:1286-96.

18. Fjorback AW, Seaman M, Gustafsen C, Mehmedbasic A, Gokool $\mathrm{S}, \mathrm{Wu} \mathrm{C}$, et al. Retromer binds the FANSHY sorting motif in SorLA to regulate amyloid precursor protein sorting and processing. J Neurosci. 2012;32:1467-80.

19. Hu F, Padukkavidana T, Vaegter CB, Brady OA, Zheng Y, Mackenzie IR, et al. Sortilin-mediated endocytosis determines levels of the frontotemporal dementia protein, progranulin. Neuron. 2010;68:654-67.

20. Wang CL, Tang FL, Peng Y, Shen CY, Mei L, Xiong WC. VPS35 regulates developing mouse hippocampal neuronal morphogenesis by promoting retrograde trafficking of BACE1. Biol Open. 2012;1:1248-57.

21. Goebbels S, Bormuth I, Bode U, Hermanson O, Schwab MH, Nave KA. Genetic targeting of principal neurons in neocortex and hippocampus of NEX-Cre mice. Genesis. 2006;44:611-21.

22. Wang C, Mei L. In utero electroporation in mice. Methods Mol Biol. 2013;1018:151-63.

23. He W, Lu Y, Qahwash I, Hu XY, Chang A, Yan R. Reticulon family members modulate BACE1 activity and amyloid-beta peptide generation. Nat Med. 2004;10:959-65. 
24. Sun D, Sun XD, Zhao L, Lee DH, Hu JX, Tang FL, et al. Neogenin, a regulator of adult hippocampal neurogenesis, prevents depressive-like behavior. Cell Death Dis. 2018;9:8.

25. Bian WJ, Miao WY, He SJ, Qiu Z, Yu X. Coordinated spine pruning and maturation mediated by inter-spine competition for cadherin/catenin complexes. Cell. 2015;162:808-22.

26. Varol D, Mildner A, Blank T, Shemer A, Barashi N, Yona S, et al. Dicer deficiency differentially impacts microglia of the developing and adult brain. Immunity. 2017;46:1030-44 e8.

27. Tian Y, Tang FL, Sun X, Wen L, Mei L, Tang BS, et al. VPS35deficiency results in an impaired AMPA receptor trafficking and decreased dendritic spine maturation. Mol Brain. 2015;8:70.

28. Switzer RC III. Application of silver degeneration stains for neurotoxicity testing. Toxicol Pathol. 2000;28:70-83.

29. Ehara A, Ueda S. Application of Fluoro-Jade C in acute and chronic neurodegeneration models: utilities and staining differences. Acta Histochem Cytochem. 2009;42:171-9.

30. Cao Q, Zhong XZ, Zou Y, Murrell-Lagnado R, Zhu MX, Dong XP. Calcium release through $\mathrm{P} 2 \mathrm{X} 4$ activates calmodulin to promote endolysosomal membrane fusion. J Cell Biol. 2015;209: 879-94.

31. Smolders K, Lombaert N, Valkenborg D, Baggerman G, Arckens L. An effective plasma membrane proteomics approach for small tissue samples. Sci Rep. 2015;5:10917.

32. Liu Y, Peng Y, Dai PG, Du QS, Mei L, Xiong WC. Differential regulation of myosin $\mathrm{X}$ movements by its cargos, DCC and neogenin. J Cell Sci. 2012;125:751-62.

33. Zhu XJ, Wang CZ, Dai PG, Xie Y, Song NN, Liu Y, et al. Myosin $\mathrm{X}$ regulates netrin receptors and functions in axonal path-finding. Nat Cell Biol. 2007;9:184-92.

34. Liu W, Tang FL, Lin S, Zhao K, Mei L, Ye J, et al. Vps35deficiency impairs SLC4A11 trafficking and promotes corneal dystrophy. PLoS ONE. 2017;12:e184906.

35. Liu W, Tang FL, Erion J, Xiao H, Ye J, Xiong WC. Vps35 haploinsufficiency results in degenerative-like deficit in mouse retinal ganglion neurons and impairment of optic nerve injuryinduced gliosis. Mol Brain. 2014;7:10.

36. Courchet J, Lewis TL Jr., Lee S, Courchet V, Liou DY, Aizawa S, et al. Terminal axon branching is regulated by the LKB1-NUAK1 kinase pathway via presynaptic mitochondrial capture. Cell. 2013;153:1510-25.

37. Gstrein T, Edwards A, Pristoupilova A, Leca I, Breuss M, PilatCarotta S, et al. Mutations in Vps15 perturb neuronal migration in mice and are associated with neurodevelopmental disease in humans. Nat Neurosci. 2018;21:207-17.

38. Lopez-Domenech G, Higgs NF, Vaccaro V, Ros H, ArancibiaCarcamo IL, MacAskill AF, et al. Loss of dendritic complexity precedes neurodegeneration in a mouse model with disrupted mitochondrial distribution in mature dendrites. Cell Rep. 2016;17:317-27.

39. Rosario M, Schuster S, Juttner R, Parthasarathy S, Tarabykin V, Birchmeier W. Neocortical dendritic complexity is controlled during development by NOMA-GAP-dependent inhibition of Cdc42 and activation of cofilin. Genes Dev. 2012;26:1743-57.

40. LoTurco J, Manent JB, Sidiqi F. New and improved tools for in utero electroporation studies of developing cerebral cortex. Cereb Cortex. 2009;19:1120-5.

41. Mattson MP, Magnus T. Ageing and neuronal vulnerability. Nat Rev Neurosci. 2006;7:278-94.

42. Paull TT, Rogakou EP, Yamazaki V, Kirchgessner CU, Gellert M, Bonner WM. A critical role for histone H2AX in recruitment of repair factors to nuclear foci after DNA damage. Curr Biol. 2000;10:886-95.

43. Lakin ND, Jackson SP. Regulation of p53 in response to DNA damage. Oncogene. 1999;18:7644-55.
44. Yan R, Shi Q, Hu X, Zhou X. Reticulon proteins: emerging players in neurodegenerative diseases. Cell Mol Life Sci. 2006;63:877-89.

45. Rademakers R, Neumann M, Mackenzie IR. Advances in understanding the molecular basis of frontotemporal dementia. Nat Rev Neurol. 2012;8:423-34.

46. Gotzl JK, Lang CM, Haass C, Capell A. Impaired protein degradation in FTLD and related disorders. Ageing Res Rev. 2016;32:122-39.

47. Kao AW, McKay A, Singh PP, Brunet A, Huang EJ. Progranulin, lysosomal regulation and neurodegenerative disease. Nat Rev Neurosci. 2017;18:325-33.

48. Van Damme P, Van Hoecke A, Lambrechts D, Vanacker P, Bogaert E, van Swieten J, et al. Progranulin functions as a neurotrophic factor to regulate neurite outgrowth and enhance neuronal survival. J Cell Biol. 2008;181:37-41.

49. Klein ZA, Takahashi H, Ma M, Stagi M, Zhou M, Lam TT, et al. Loss of TMEM106B ameliorates lysosomal and frontotemporal dementia-related phenotypes in progranulin-deficient mice. Neuron. 2017;95:281-96 e6.

50. Ward ME, Chen R, Huang HY, Ludwig C, Telpoukhovskaia M, Taubes A, et al. Individuals with progranulin haploinsufficiency exhibit features of neuronal ceroid lipofuscinosis. Sci Transl Med. 2017;9:eaah5642.

51. Canuel M, Lefrancois S, Zeng J, Morales CR. AP-1 and retromer play opposite roles in the trafficking of sortilin between the Golgi apparatus and the lysosomes. Biochem Biophys Res Commun. 2008;366:724-30

52. Seaman MN. Cargo-selective endosomal sorting for retrieval to the Golgi requires retromer. J Cell Biol. 2004;165:111-22.

53. Rost BR, Schneider F, Grauel MK, Wozny C, Bentz C, Blessing A, et al. Optogenetic acidification of synaptic vesicles and lysosomes. Nat Neurosci. 2015;18:1845-52.

54. Sharma J, di Ronza A, Lotfi P, Sardiello M. Lysosomes and brain health. Annu Rev Neurosci. 2018;41:255-76.

55. Small SA, Petsko GA. Retromer in Alzheimer disease, Parkinson disease and other neurological disorders. Nat Rev Neurosci. 2015;16:126-32.

56. Menzies FM, Fleming A, Rubinsztein DC. Compromised autophagy and neurodegenerative diseases. Nat Rev Neurosci. 2015;16:345-57.

57. Frake RA, Ricketts T, Menzies FM, Rubinsztein DC. Autophagy and neurodegeneration. J Clin Investig. 2015;125:65-74.

58. Arighi CN, Hartnell LM, Aguilar RC, Haft CR, Bonifacino JS. Role of the mammalian retromer in sorting of the cationindependent mannose 6-phosphate receptor. J Cell Biol. 2004;165:123-33.

59. Cui Y, Carosi JM, Yang Z, Ariotti N, Kerr MC, Parton RG, et al. Retromer has a selective function in cargo sorting via endosome transport carriers. J Cell Biol. 2019;218:615-31.

60. Maruzs T, Lorincz P, Szatmari Z, Szeplaki S, Sandor Z, Lakatos $Z$, et al. Retromer ensures the degradation of autophagic cargo by maintaining lysosome function in Drosophila. Traffic. 2015;16:1088-107.

61. Zavodszky E, Seaman MN, Moreau K, Jimenez-Sanchez M, Breusegem SY, Harbour ME, et al. Mutation in VPS35 associated with Parkinson's disease impairs WASH complex association and inhibits autophagy. Nat Commun. 2014;5:3828.

62. Lu X, Meima ME, Nelson JK, Sorrentino V, Loregger A, Scheij S, et al. Identification of the (Pro)renin receptor as a novel regulator of low-density lipoprotein metabolism. Circ Res. 2016;118:222-9.

63. Mattson MP. Apoptosis in neurodegenerative disorders. Nat Rev Mol Cell Biol. 2000;1:120-9.

64. Ekshyyan O, Aw TY. Apoptosis: a key in neurodegenerative disorders. Curr Neurovasc Res. 2004;1:355-71. 
65. Su JH, Nichol KE, Sitch T, Sheu P, Chubb C, Miller BL, et al. DNA damage and activated caspase- 3 expression in neurons and astrocytes: evidence for apoptosis in frontotemporal dementia. Exp Neurol. 2000;163:9-19.

66. Bennion Callister J, Pickering-Brown SM. Pathogenesis/genetics of frontotemporal dementia and how it relates to ALS. Exp Neurol. 2014;262:84-90.

67. Simon HU, Haj-Yehia A, Levi-Schaffer F. Role of reactive oxygen species (ROS) in apoptosis induction. Apoptosis. 2000;5:415-8.

68. Farmer T, O'Neill KL, Naslavsky N, Luo X, Caplan S. Retromer facilitates the localization of Bcl-xL to the mitochondrial outer membrane. Mol Biol Cell. 2019;30:1138-46.

69. Chen D, Xiao H, Zhang K, Wang B, Gao Z, Jian Y, et al. Retromer is required for apoptotic cell clearance by phagocytic receptor recycling. Science. 2010;327:1261-4.
70. Marino G, Niso-Santano M, Baehrecke EH, Kroemer G. Selfconsumption: the interplay of autophagy and apoptosis. Nat Rev Mol Cell Bio. 2014;15:81-94.

71. Gump JM, Thorburn A. Autophagy and apoptosis: what is the connection? Trends Cell Biol. 2011;21:387-92.

72. Temkin P, Morishita W, Goswami D, Arendt K, Chen L, Malenka $\mathrm{R}$. The retromer supports AMPA receptor trafficking during LTP. Neuron. 2017;94:74-82 e5.

73. Choy RW, Park M, Temkin P, Herring BE, Marley A, Nicoll RA, et al. Retromer mediates a discrete route of local membrane delivery to dendrites. Neuron. 2014;82:55-62.

74. Munsie LN, Milnerwood AJ, Seibler P, Beccano-Kelly DA, Tatarnikov I, Khinda J, et al. Retromer-dependent neurotransmitter receptor trafficking to synapses is altered by the Parkinson's disease VPS35 mutation p.D620N. Hum Mol Genet. 2015;24:1691-703. 